\title{
Bariatric and Metabolic Surgery
}

\author{
Chih Kun Huang, Sir Emmanuel S. Astudillo, \\ Prasad M. Bhukebag and Khan Wei Chan
}

Additional information is available at the end of the chapter

http://dx.doi.org/10.5772/107288

\begin{abstract}
Obesity as a global epidemic has rapidly increased in incidence in the recent few decades and represents one of the biggest public health challenges. Obesity plays a major risk for various diseases such as cardiovascular disease (CVD), diabetes mellitus (DM), hypercholesterolemia, osteoarthritis and some form of cancers. Bariatric and metabolic surgery provides the best solution for obesity and its associated comorbidities. This chapter will discuss in detail the commonly performed bariatric and metabolic surgeries.
\end{abstract}

Keywords: bariatric surgery, metabolic surgery, Roux-en-Y gastric bypass, sleeve gastrectomy, duodenojejunal bypass, proximal jejunal bypass, obesity, type 2 diabetes

\section{Introduction}

Bariatric surgery has been established to be the most effective treatment for morbid obesity, producing sustained and durable weight loss with improvement or remission of comorbidities and longer life [1]. The procedures undertaken to treat morbid obesity have changed over a period of time and recently newer procedures are being developed with lesser morbidity and mortality and better results. The resolution of comorbidities such as type 2 diabetes mellitus (T2DM), hypertension and others after bariatric surgery gave impetus to the concept of metabolic surgery. Metabolic surgery has become increasingly effective and accepted option for patients with T2DM [2]. This chapter will describe in detail two most commonly performed procedures, laparoscopic sleeve gastrectomy (LSG) and laparoscopic Roux-en-Y gastric bypass (LRYGB). Part of the chapter also details the sleeve gastrectomy-based procedures which include sleeve gastrectomy with loop duodenojejunal bypass (LDJB-SG) and sleeve gastrectomy with proximal jejunal bypass (SG-PJB). 


\section{Laparoscopic sleeve gastrectomy}

Laparoscopic Sleeve Gastrectomy (LSG) or "sleeve" is now one of the most commonly performed bariatric procedures worldwide. It is a purely restrictive procedure without intestinal bypass. In 1999, Michel Gagner and his team performed the first laparoscopic duodenal switch on the porcine model and found it to be feasible. Laparoscopic duodenal switch was later performed in morbid obese patients and found to have higher morbidity in higher BMI patients. Staged procedure was then undertaken to reduce the mortality and morbidity with laparoscopic sleeve gastrectomy as the first stage of a two-stage duodenal switch. However, the initial weight loss after LSG alone was found to be adequate and maintained over a period of time. The second stage of surgery was deferred or was not required at all and patients maintained good weight loss with just the first stage. After this success and some modifications to the procedure, sleeve gastrectomy was established as an effective stand-alone bariatric procedure.

Apart from restriction of size of stomach, reduction in the "hunger hormone" ghrelin, after LSG decreases appetite. Advantages of LSG include lower operative complexity, relative safety and maintenance of pylorus and easy convertibility to other procedures. Also, since there are no bowel bypass problems of internal herniation, small bowel obstruction, micronutrient deficiencies and malnutrition will not be encountered.

The disadvantages of the procedure include irreversibility, possibility of staple line leak, bleeding and stricture of gastric tube. The incidence of gastroesophageal reflux has also been found to be increased in some patients undergoing this procedure.

\subsection{Indications and contraindications}

Indications for this procedure include BMI $\geq 40 \mathrm{~kg} / \mathrm{m}^{2}$ without comorbidities or $\geq 35 \mathrm{~kg} / \mathrm{m}^{2}$ with comorbidities. This can also be offered as a first stage of biliopancreatic diversion in patients with $\mathrm{BMI}>50 \mathrm{~kg} / \mathrm{m}^{2}$. Contraindications include chronic alcoholism, drug and substance abuse, major psychiatric disorder, severe gastroesophageal reflux disease and chronic duodenal ulcer.

\subsection{Preoperative work up and preparation}

Comprehensive preoperative workup for sleeve gastrectomy is the same as that of the other bariatric procedures. Patients are admitted the day prior to the procedure when they are started on clear liquid diet. Single dose of prophylactic antibiotic and proton pump inhibitor are given an hour prior to surgery. Low molecular-weight heparin (LMWH) is prescribed to lower the incidence of deep vein thrombosis (DVT) and other thromboembolic events, till the patient is ambulated.

\subsection{Operative technique}

\subsubsection{Positioning}

Patient is positioned flat on the operating table with both upper extremities extended out. Patient is secured at the chest and thighs using straps and a foot board in preparation for a steep reverse Trendelenburg position. The surgeon and the scrub nurse both stand on the patient's right while the first assistant and the cameraman stand on the patient's left. 


\subsubsection{Port placement}

A three-four ports technique is utilized for this procedure as shown in Figure 1. Pneumoperitoneum is created by Veress needle or optical trocar is used at the first port. The first port is about three finger breadths to the left of umbilicus, at about $20 \mathrm{~cm}$ from the xiphisternum. The second $15 \mathrm{~mm}$ port is inserted at supraumbilical site. Another $5 \mathrm{~mm}$ trocar is inserted in the right midclavicular line below the costal margin, for surgeon's left hand, such that the falciform ligament does not cause difficulty in dissection of the hiatus. Lastly an optional $5 \mathrm{~mm}$ assistant port can be placed in the left anterior axillary line just below the costal margin.

\subsubsection{Placement of liver suspension tape}

To elevate the central and left lobe of the liver, a liver suspension tape (LST) was designed. Two pieces of Jackson-Pratt (JP) drain measuring $2 \mathrm{~cm}$ each were cut and fixed with 2/0 polypropylene suture (Ethicon W8400 2-0 Prolene blue $70 \mathrm{~mm}$ round bodied). The lateral segment of the left lobe was suspended using the tape by passing the needle through the liver, out through the anterior abdominal wall and secured with clamps. Another LST was inserted in the medial aspect of left lobe of liver to retract the liver completely.

The LSTs should not be placed more than $2 \mathrm{~cm}$ away from the liver edge to achieve optimal retraction and prevent major bile duct or vessel injury (Figure 2). Alternatively, a Nathanson

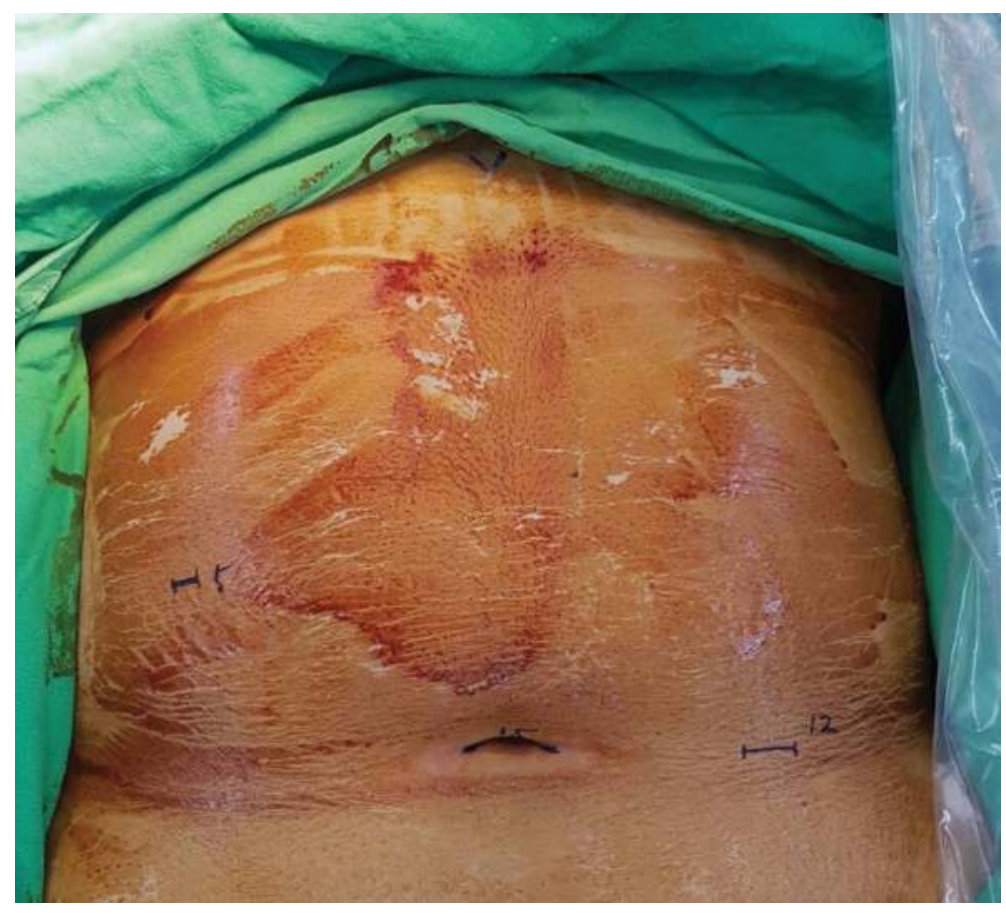

Figure 1. Port placement for LSG. 
liver retractor (C-NLRS-1001 Cook Medical) may be used in order to achieve good exposure of the entire length of the stomach and duodenum. This may be placed using a $5 \mathrm{~mm}$ port inserted in the subxiphoid area.

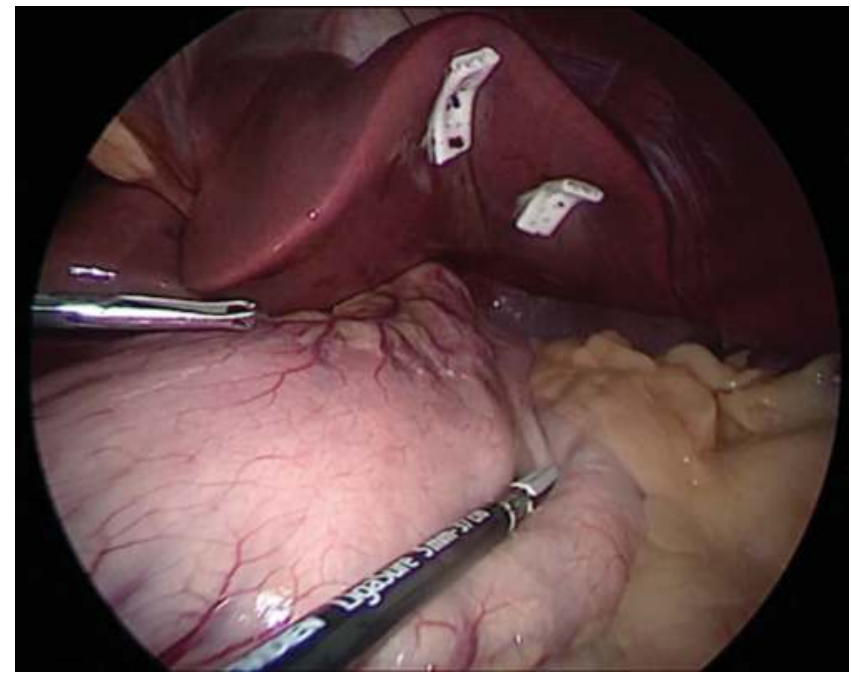

Figure 2. Retraction of left lobe of liver with liver suspension tape.

\subsubsection{Mobilization of the gastrocolic ligament}

Dissection of the gastrocolic ligament close to the greater curvature of the stomach commences at a point opposite the incisura angularis. A window in the lesser sac is created and dissection is started using a vessel sealing energy device (Ligasure ${ }^{\mathrm{TM}}$ vessel sealing device by Covidien-Medtronic or Harmonic Ace by Ethicon). By staying very close to the gastric wall, the entire gastrocolic ligament is detached up to the angle of His taking care at the area of the short gastric vessels at the splenic hilum (Figures 3 and 4). Care should be taken not to injure left gastric vessels.

\subsubsection{Dissection at the angle of His}

Fundus of the stomach must be mobilized from its adhesions and completely resected. A very useful anatomical landmark is to expose the left crus of the hiatus. Dissection of the left crus is terminated once the left phrenoesophageal ligament has been completely visualized (Figure 5). The posterior attachments of the fundus to the anterior border of the pancreas are completely mobilized to properly identify and preserve the left gastric vessels (Figure 6).

The anterior portion of the gastroesophageal fat pad (Belsey's pad of fat) is then dissected in order to expose half of the anterior portion of the GE junction. Care is taken not to completely devascularize this fat pad which may increase the rate of leak at the GE junction. 


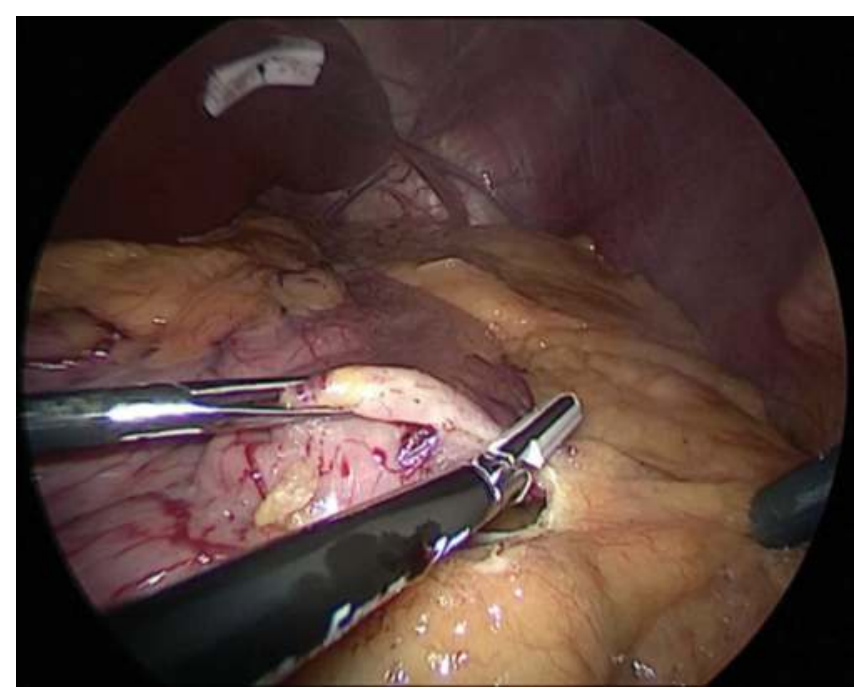

Figure 3. Start of dissection by creating a window in the lesser sac.

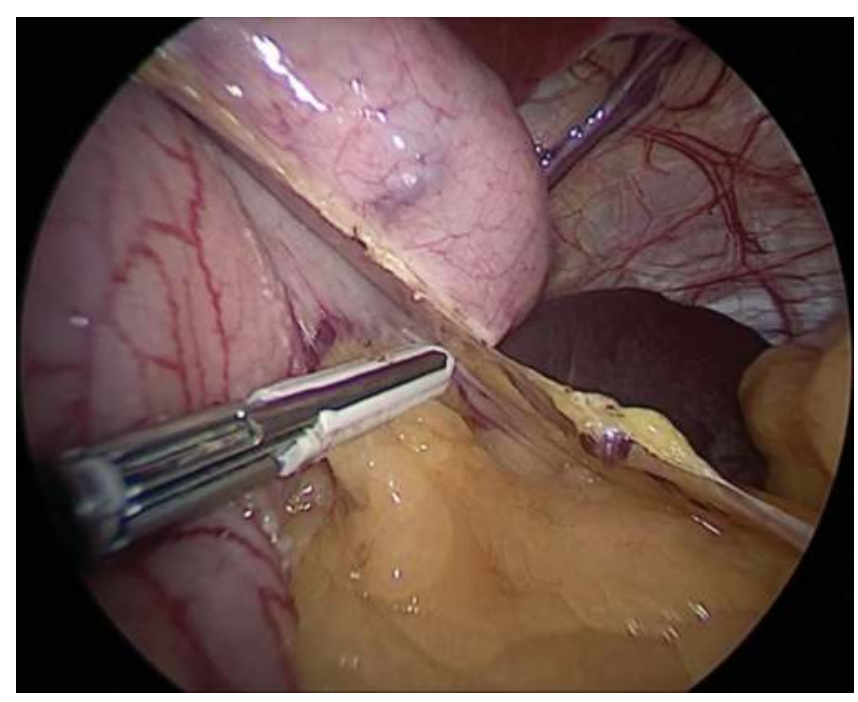

Figure 4. Dissection of the gastrocolic ligament at short gastric vessels near splenic hilum.

\subsubsection{Dissection of the caudal part of the gastrocolic ligament}

The gastrocolic ligament at the area of the right gastroepiploic vessel is dissected caudally till the distance of $4 \mathrm{~cm}$ from the pylorus. Dense adhesions around the prepyloric area may be encountered and should be taken down individually (Figure 7). 


\subsubsection{Sleeve gastrectomy}

Prior to the creation of the actual sleeve, make sure that the entire stomach is laid down flat. At this point, the anesthesiologist inserts an orogastric tube with its tip positioned by the surgeon under direct visual guidance. Using an L-hook dissector (STORZ 37370DL Monopolar Dissecting L-Hook Cannula), marking on the anterior surface of the stomach is done which

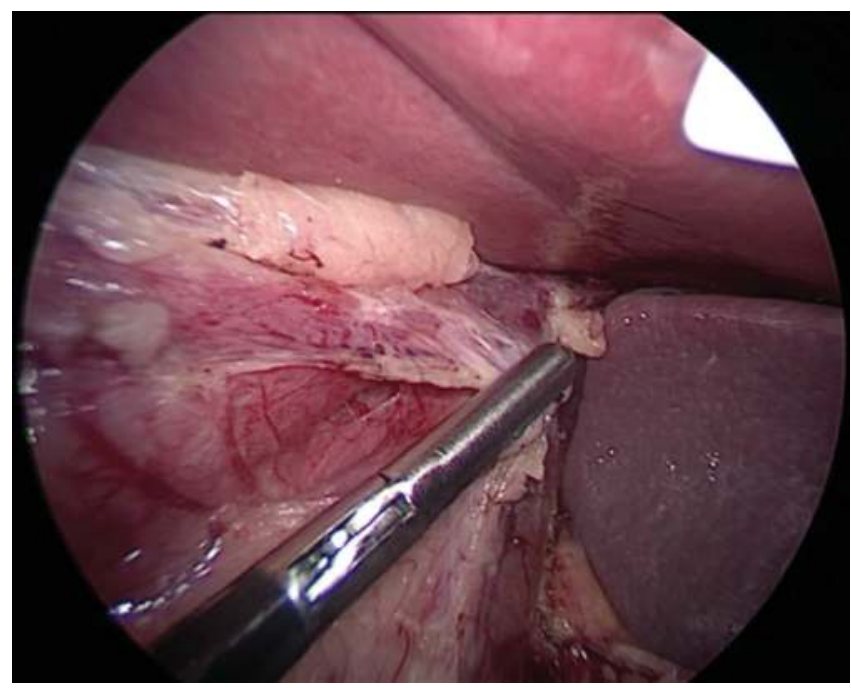

Figure 5. Dissection at the angle of His exposing the left crus and phrenoesophageal ligament.

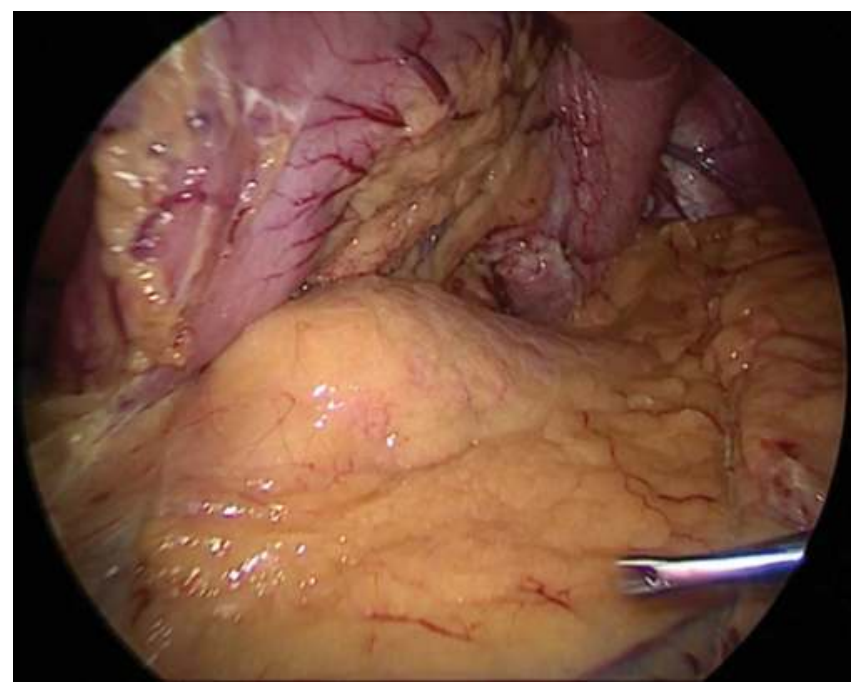

Figure 6. Complete mobilization of the posterior attachments of the fundus and anterior border of the pancreas. 
will serve as a guide for the surgeon to place the endostapler with $38 \mathrm{Fr}$ orogastric tube as stent (Figure 8). For the first staple firing, the stapling device is placed $4 \mathrm{~cm}$ away from the pylorus and 2.5-3 cm away from the incisura angularis in order to prevent narrowing (Figure 9). A black $60 \mathrm{~mm}$ reload (EGIA60AXT Endo GIA $60 \mathrm{~mm}$ articulating extra-thick reload with Tri-Staple Technology) or a green $60 \mathrm{~mm}$ green reload (ECR60G Echelon Endopath stapler reload cartridges by Ethicon) using manual or powered device is used. Successive firings

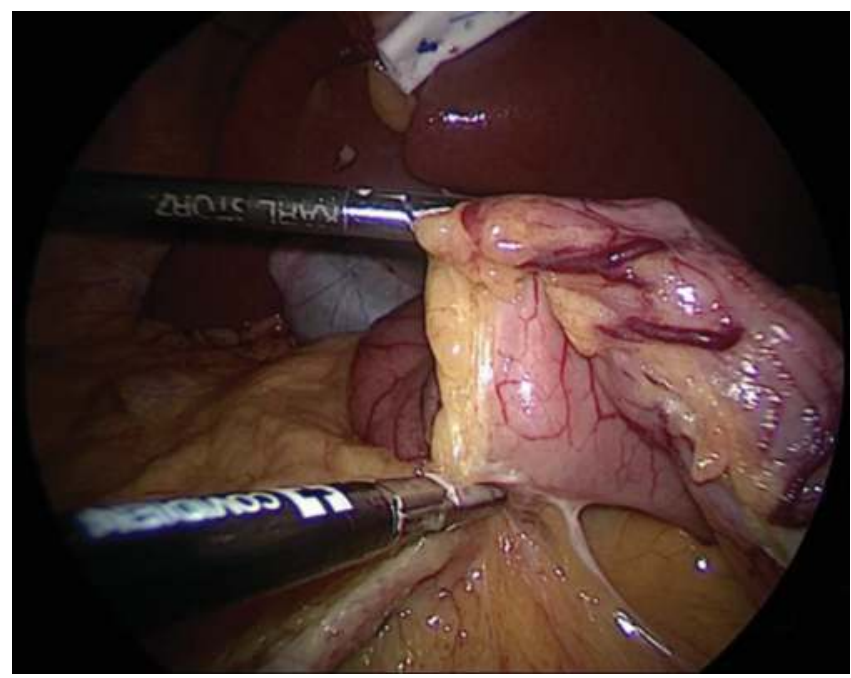

Figure 7. Dissection of the gastrocolic ligament toward the pylorus.

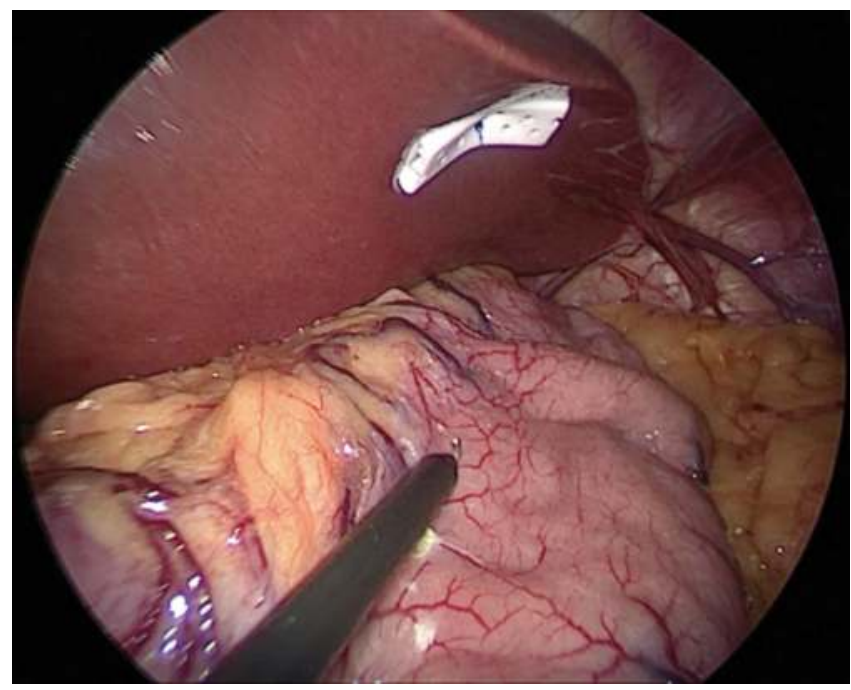

Figure 8. Marking of the transection line along the anterior surface of the stomach. 
of the stapling device toward the gastric fundus are done using either a $60 \mathrm{~mm}$ purple cartridge (EGIA60AMT Endo GIA $60 \mathrm{~mm}$ articulating medium/thick reload with Tri-Staple Technology) or a $60 \mathrm{~mm}$ blue cartridge (ETHECR60B Echelon Endopath stapler reload cartridges by Ethicon) (Figure 10). Commencing after placement of the second stapler, loose migratory crotch staples must be removed in order to prevent improper formation of the

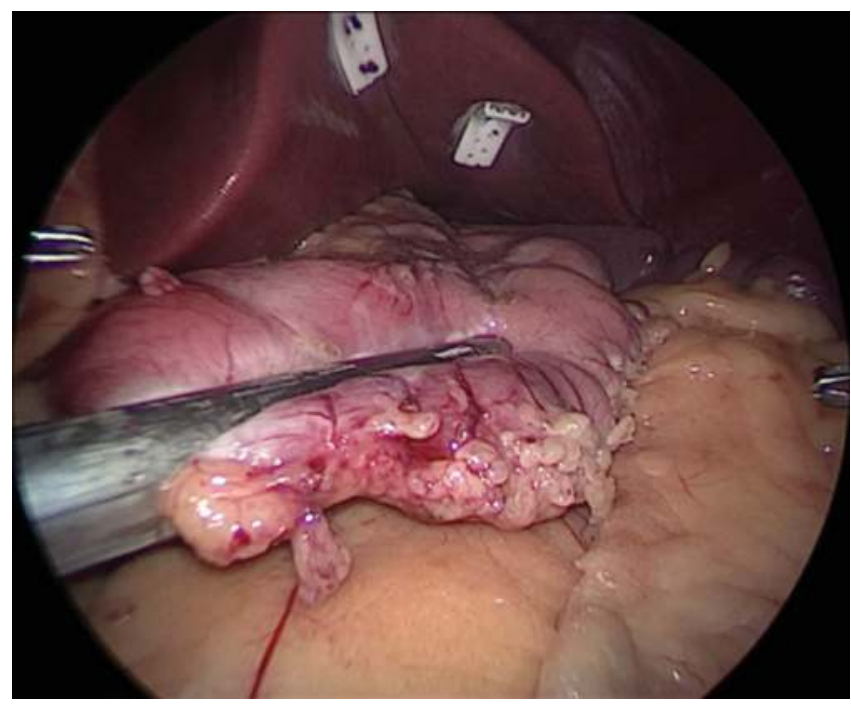

Figure 9. First staple firing $4 \mathrm{~cm}$ from pylorus and $2.5-3 \mathrm{~cm}$ away from the incisura.

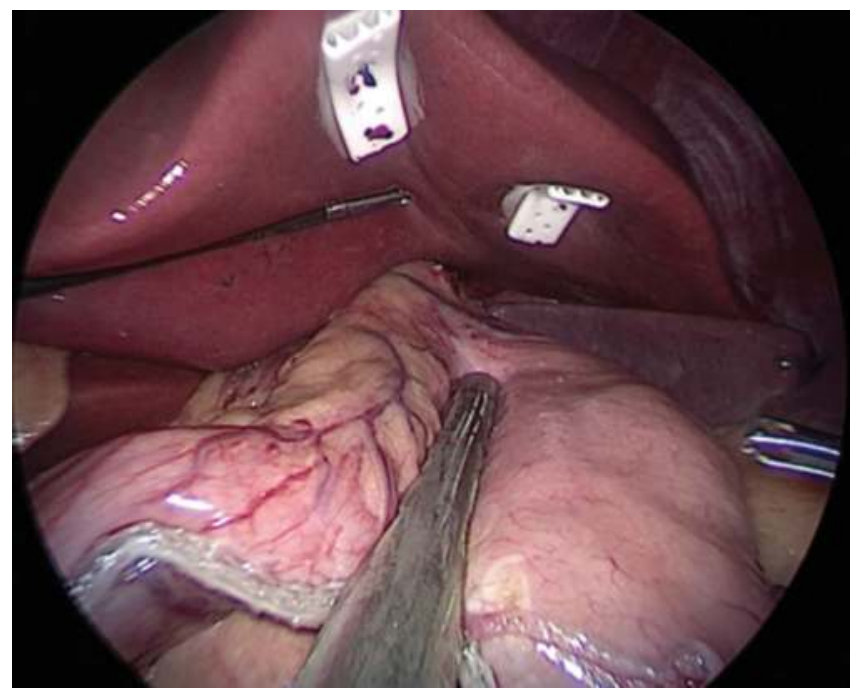

Figure 10. Successive firings of the staple device toward the fundus. 
suture line (Figure 11). Constant communication with the anesthesiologist is of paramount importance, inserting and withdrawing the orogastric tube before and after application of each stapler to make sure the bougie is not stapled into the remnant stomach. It is also highly recommended to check the posterior aspect of the stomach before each firing of the device in order to avoid twisting and in-folding of tissues. The entire fundus, including its posterior

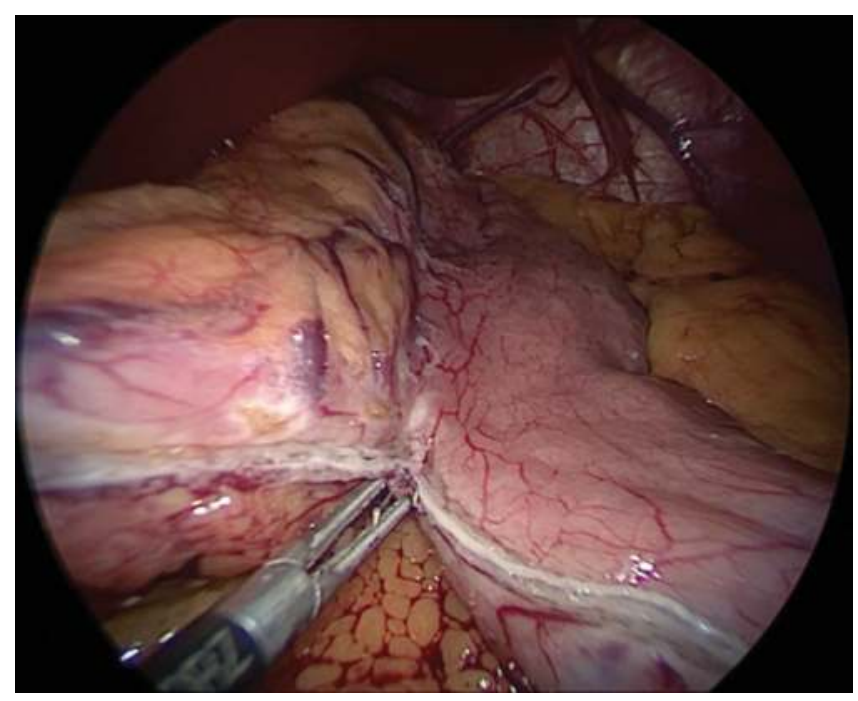

Figure 11. Removal of migratory crotch staples.

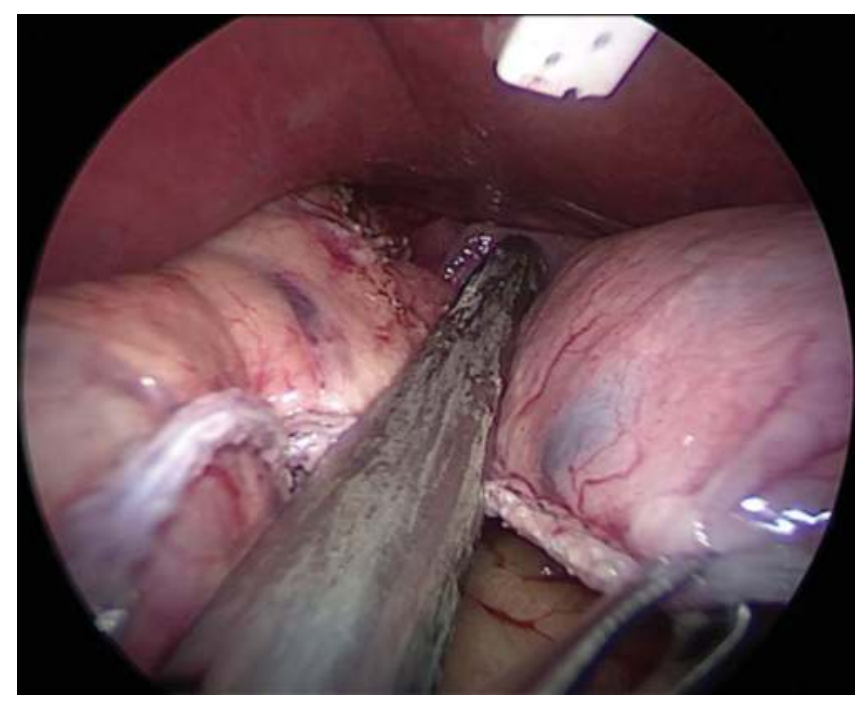

Figure 12. Staple firing $1 \mathrm{~cm}$ away from the GE junction. 
wall, is to be included in the resected part. Before firing of the last stapler, always verify that the stapler is placed not less than $1 \mathrm{~cm}$ from the GE junction and that both tips of the stapler are always in sight (Figure 12).

\subsubsection{Hemostasis}

The entire length of the staple line should be inspected for any bleeding. Bleeding may be controlled using electrocautery, clips or over sewing. No buttressing material is placed over the staple line.

\subsubsection{Fixing the tube}

One or two fixing stitches are taken between the stapled sleeve and the retroperitoneal fat to prevent rotation of the sleeve tube.

\subsubsection{Specimen extraction}

The LSTs are removed and the entry and exit points on the liver are cauterized to achieve hemostasis. Specimen is then extracted through the umbilical port and closure of the rectus sheath done with 2-0 Vicryl. Subcutaneous layers of all ports closed with 3-0 Vicryl and skin closed with interrupted subcuticular stitches. Dermabond is applied and dressing done.

\subsubsection{Surgical outcomes}

The mechanism of action of LSG appears to be by the restriction of the volume of stomach and the removal of ghrelin-producing fundus [3]. The decreased ghrelin leads to early satiety and decreased hunger. Another mechanism is increased gastric emptying which combined with decreased gastric acid secretion causes incomplete digestion [4]. Increased gastric emptying is associated with higher levels of glucagon-like-peptide-1 (GLP-1), a glucose-regulating insulin-enhancing agent, which has been linked to weight loss and resolution of type 2 diabetes mellitus [5].

In a retrospective study, the EWL after 3 and 6 years follow-up of LSG was 72.8 and $57.3 \%$, respectively [4]. Gustavo et al. in their long-term study showed a mean \%EBMIL (percentage of excess BMI Loss) of 82.4, 75.9 and 62.5 and \% TWL (percentage of total weight loss) of 28.5, 25.8 and 21 at 3,6 and 11+ years of follow-up, respectively [6].

\section{Sleeve-based procedures}

One of the arguments against LSG is that its weight loss as well as its efficacy in diabetes remission is inferior and not sustainable compared to RYGB. This is owed to the fact that LSG do not own the content of bowel bypass. Laparoscopic loop duodenojejunal bypass with sleeve gastrectomy (LDJB-SG) and sleeve gastrectomy with proximal jejunal bypass (SG-PJB) are a combination of both a restrictive and malabsorptive procedures (Figure 13 and 28). First described by Huang et al. in 2011, LDJB-SG has a lower incidence of dumping syndrome and marginal ulcer, both commonly experienced in RYGB, due to an intact pylorus which acts as 


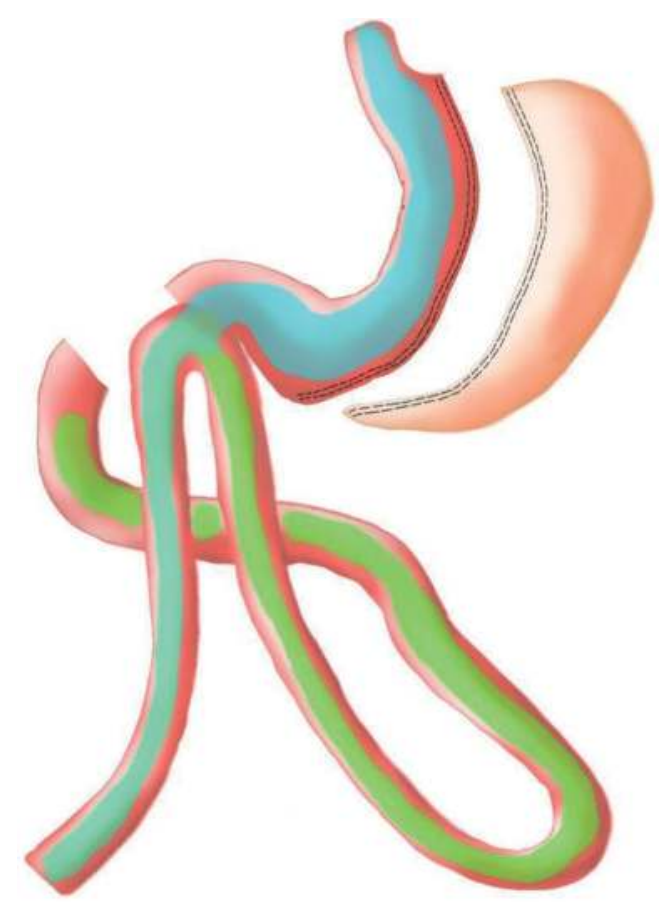

Figure 13. Schematic diagram of LDJB-SG.

a sphincter mechanism and a creates a neutral condition from mixture of gastric acid, pancreatic juice and bile around the duodenojejunal anastomosis [7]. SG-PJB was designed by Alamo et al. in 2005 and later revised in 2009 [9, 10]. Surgical results of weight loss and comorbidity resolution were comparable to Roux-en-Y gastric bypass in his reports. Both LDJB-SG and SGPJB can be an alternative operation that could potentially lessen the complications associated with the conventional gastric bypass.

\subsection{Loop DuodenoJejunal Bypass with Sleeve Gastrectomy}

\subsubsection{Indications and Contraindications}

Indication for this procedure includes BMI $\geq 40 \mathrm{~kg} / \mathrm{m}^{2}$ without comorbidities or $\geq 35 \mathrm{~kg} / \mathrm{m}^{2}$. This can also be offered as a surgical treatment for poorly controlled type 2 diabetes in Asian patients with $\mathrm{BMI} \geq 32.5 \mathrm{~kg} / \mathrm{m}^{2}$ without comorbidities or $>27.5 \mathrm{~kg} / \mathrm{m}^{2}$ with comorbidities. Contraindications include chronic alcoholism, drug and substance abuse, major psychiatric disorder, severe gastroesophageal reflux disease and chronic duodenal ulcer [8].

\subsubsection{Preoperative work up and preparation}

Comprehensive preoperative workup for DJB-SG is the same as that of the other bariatric procedures. Patients are admitted the day prior to the procedure were they are started on clear liquid diet. Single dose of prophylactic antibiotic and proton pump inhibitor are given 
an hour prior to surgery. Low molecular-weight heparin (LMWH) is prescribed to lower the incidence of deep vein thrombosis (DVT) and other thromboembolic events.

\subsubsection{Positioning}

Patient is positioned flat on the operating table with both upper extremities extended out. Patient is secured at the chest and thighs using straps and a foot board in preparation for a steep reverse Trendelenburg position. The surgeon and the scrub nurse both stand on the patient's right while the first assistant and the cameraman stand on the patient's left.

\subsubsection{Operative Technique}

\subsubsection{Port placement}

Five-port technique is utilized for this procedure as shown in Figure 14. A $12 \mathrm{~mm}$ optical port on the left of umbilicus is placed after abdominal cavity is entered using the closed Veress technique or via an optical trocar. One $15 \mathrm{~mm}$ port is placed in immediate supraumbilical region and another $12 \mathrm{~mm}$ port is placed to the right of umbilicus mirroring the one on the left, both of which serve as the surgeon's right hand working ports. A $5 \mathrm{~mm}$ trocar is placed in the right midclavicular line below the costal margin for the surgeon's left hand while another 5 $\mathrm{mm}$ trocar is placed in the midclavicular line below the left subcostal margin for the assistant.

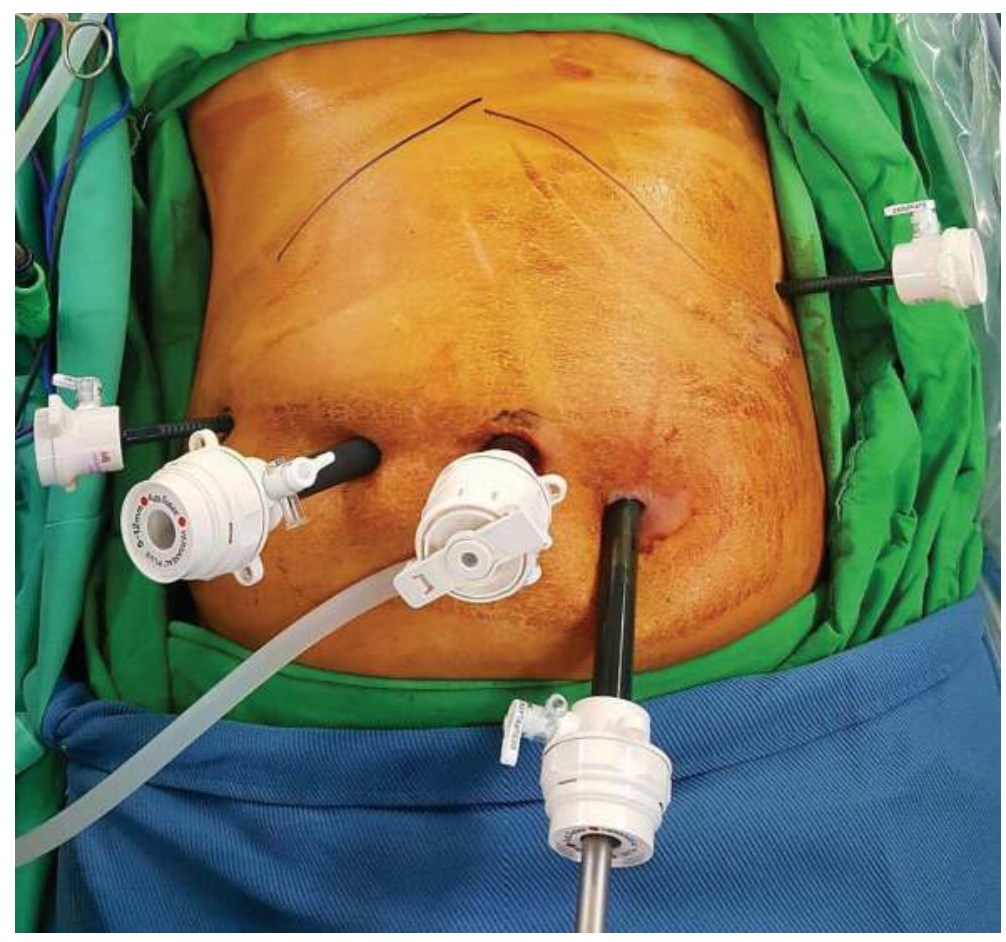

Figure 14. Port placement for LDJB-SG. 


\subsubsection{Placement of liver suspension tape}

An LST previously discussed earlier in this chapter is utilized. However, instead of using a $2.5 \mathrm{~cm}$ cut JP drain, $5 \mathrm{~cm}$ is used for the second tape in order to lift both the lobes of the liver along with the falciform ligament away from the field (Figure 15).

\subsubsection{Sleeve gastrectomy}

The formation of the gastric sleeve tube is done in a similar way as described earlier in this chapter.

\subsubsection{Duodenal transection}

Proper exposure of the entire length of the stomach and first part of the duodenum is critical to the success of this procedure. After sleeve gastrectomy has been performed, the next step is to dissect the first part of the duodenum. A counter traction suture is placed at the distal end of the sleeved stomach to visualize the first part of the duodenum (Figure 16). At a distance approximately $2 \mathrm{~cm}$ distal to the pylorus, a tunnel is created posterior to the duodenal wall and just anterior to the gastroduodenal artery using a combination of a blunt dissector and Flexlap Gold Finger retractor (Flexlap Gold Finger Retractor by Ethicon Endo Surgery, USA) as seen in Figures 17 and 18. A tape is then passed in the tunnel behind the duodenum and lifted laterally and downwards to serve as a traction while inserting the endostapler (Figure 19). Using a $45 \mathrm{~mm}$ curved tip articulating tan reload (EGIA45CTAVM Endo GIA $45 \mathrm{~mm}$ Curved Tip articulating vascular/medium reload with Tri-Staple ${ }^{\mathrm{TM}}$ Technology), the first part of the duodenum is transected. Care is taken to avoid injury to the common bile duct (CBD), pancreas and major vessels in the area (Figure 20) [8].

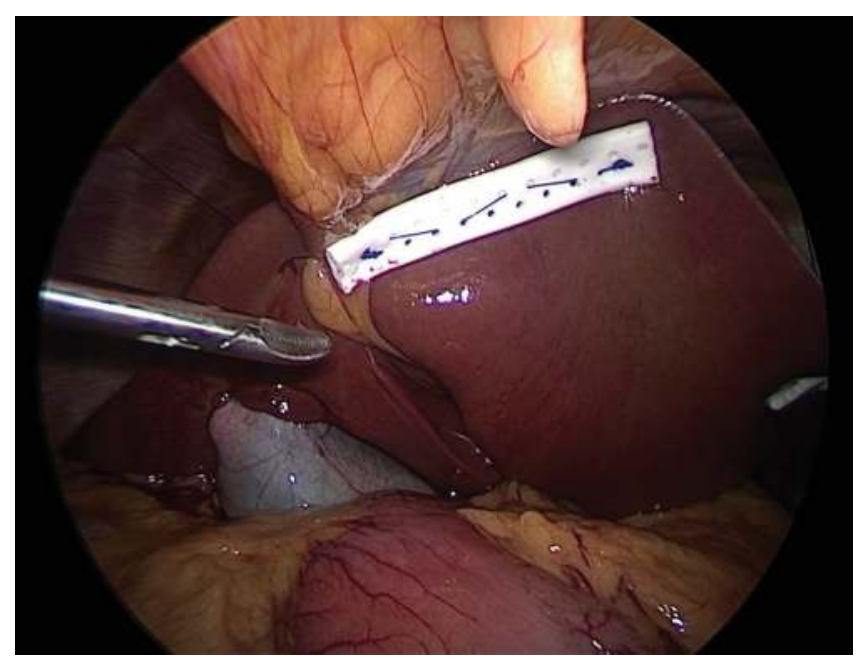

Figure 15. Liver suspension for both lobes. 


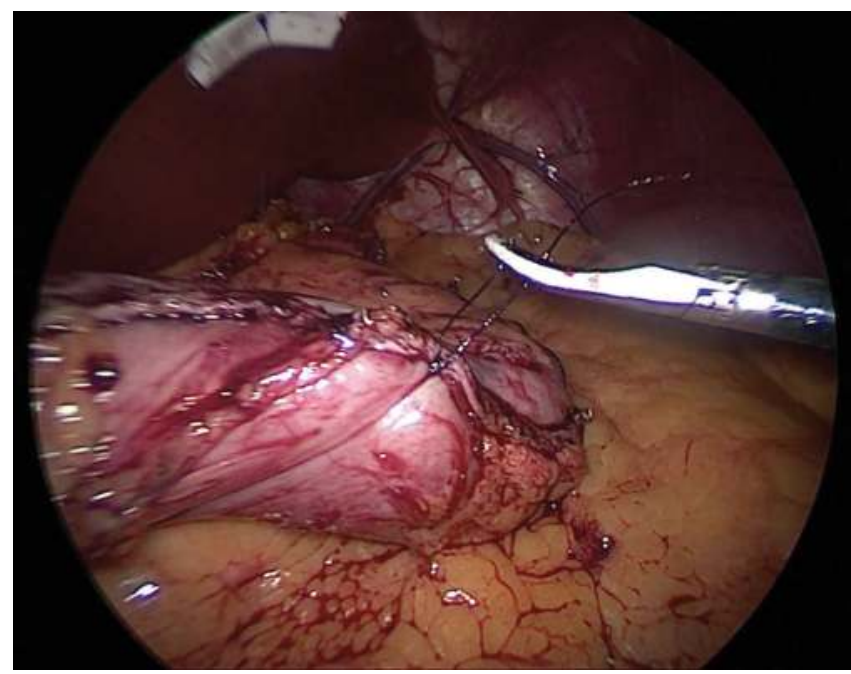

Figure 16. Counter traction suture.

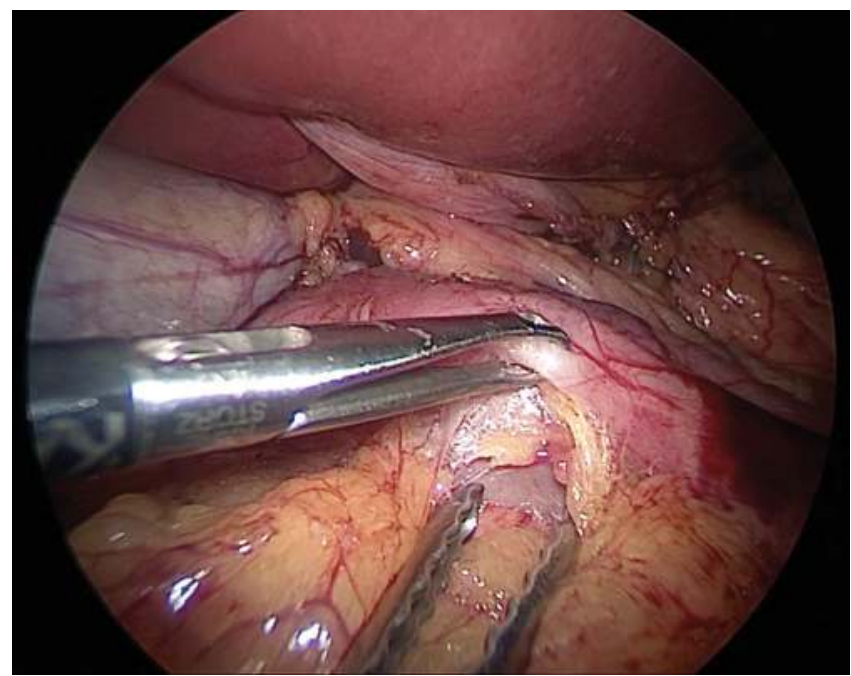

Figure 17. Retroduodenal tunnel.

\subsubsection{Duodenojejunostomy}

The ligament of Treitz is identified as it exits at the root of the transverse mesocolon. A length of jejunum is measured for $200-300 \mathrm{~cm}$ starting from the ligament of Treitz. The loop of jejunum is pulled up and a stay suture is placed between the loop of jejunum and pylorus. An enterotomy of $1.5 \mathrm{~cm}$ is placed obliquely in the first part of the duodenum and antimesenteric 


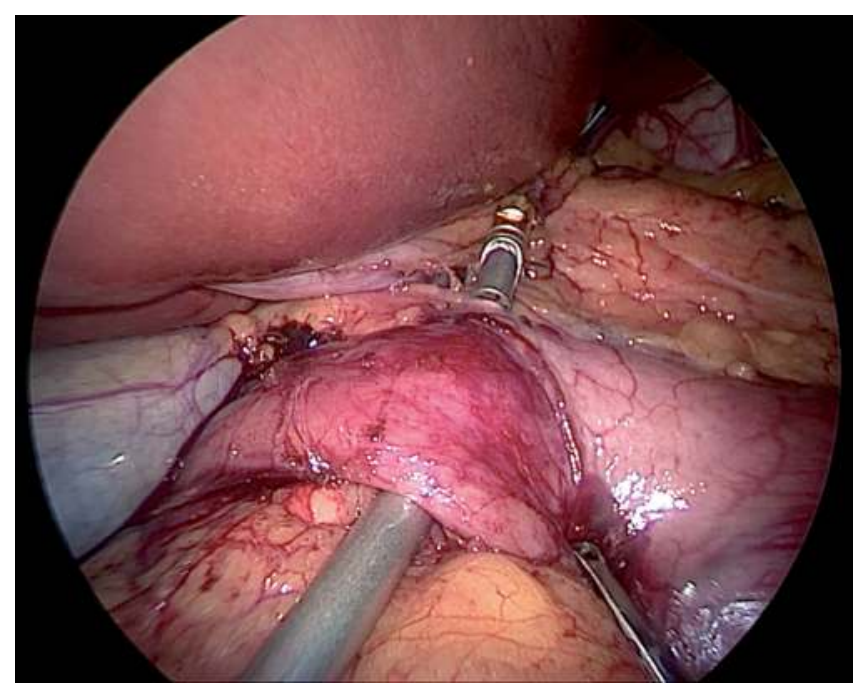

Figure 18. Retroduodenal tunnel passing through the lesser sac using the Goldfinger retractor.

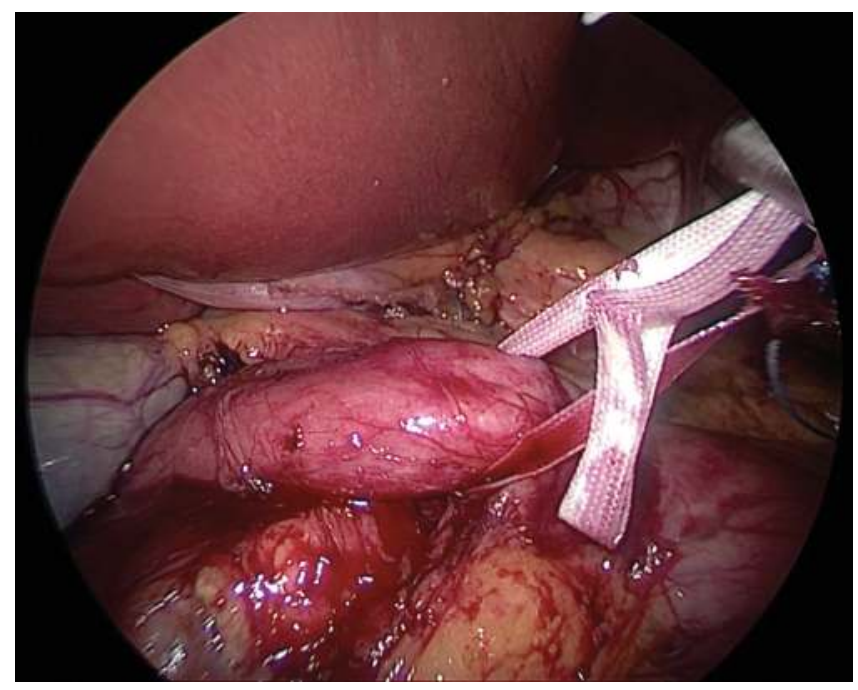

Figure 19. Lifting and retraction of the 1st portion of the duodenum using a tape.

side of jejunum (Figure 21 and 22). A completely hand-sewn side to side duodenojejunal anastomosis in a running fashion is created using a 3-0 absorbable glyconate monofilament suture (3-0 B I BRAUN MONOSYN ${ }^{\text {TM }}$ UNDYED 28" HR26 TAPER) (Figures 23-25). Then an antitorsion suture between antrum and jejunum is placed, around $4 \mathrm{~cm}$ proximal to the D-J anastomosis [8]. 


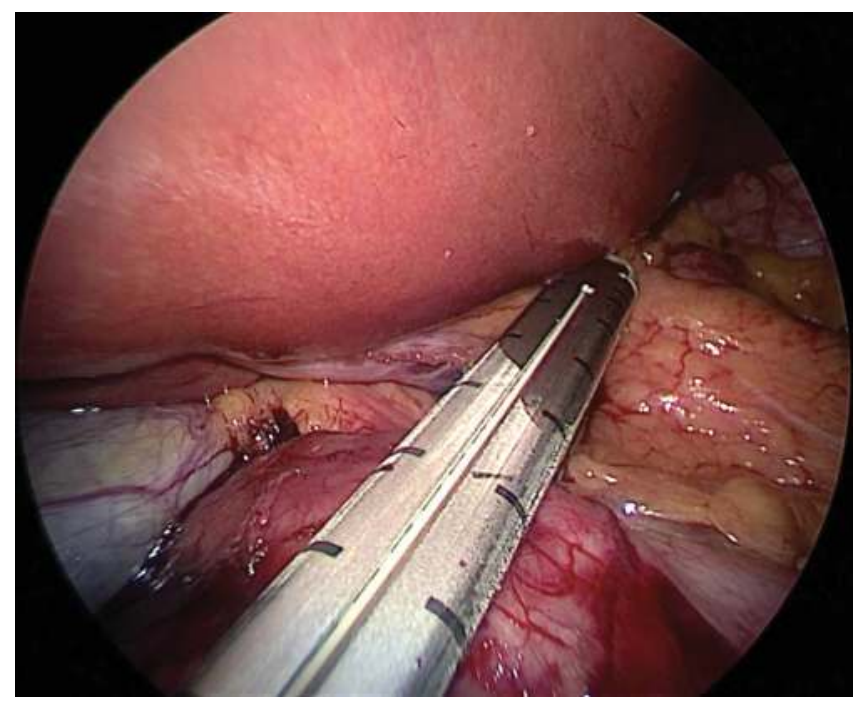

Figure 20. Duodenal transection.

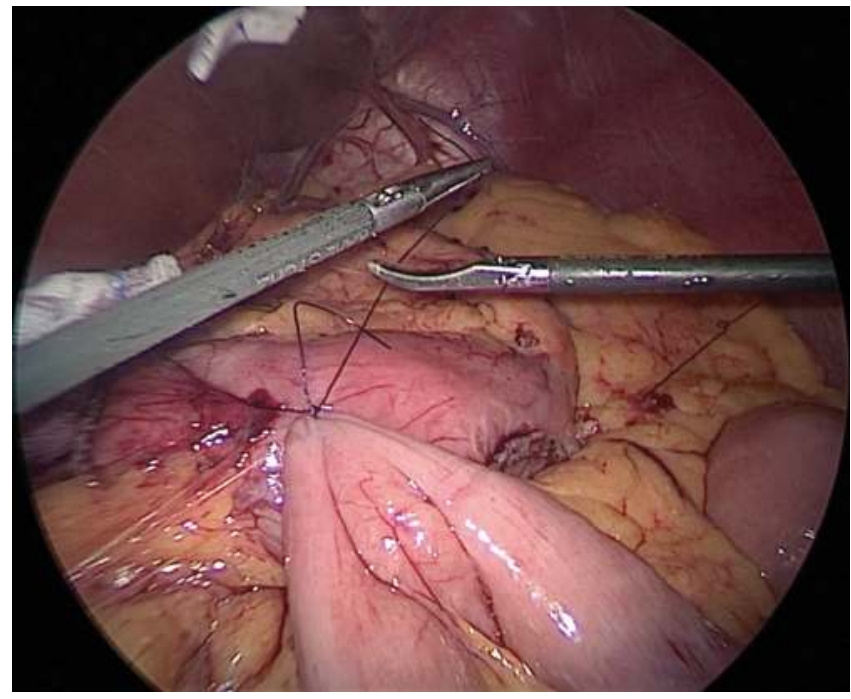

Figure 21. Stay suture between sleeved stomach and jejunum.

\subsubsection{Closure of mesenteric defect}

Peterson's mesenteric defect is closed in a continuous running technique using 2-0 nonabsorbable polyester suture (W6977 Ethibond Excel ${ }^{\mathrm{TM}}$ Polyester suture) (Figure 26). A Jackson Pratt drain is placed under the entire length of stomach tube and duodenojejunal anastomosis (Figure 27). 


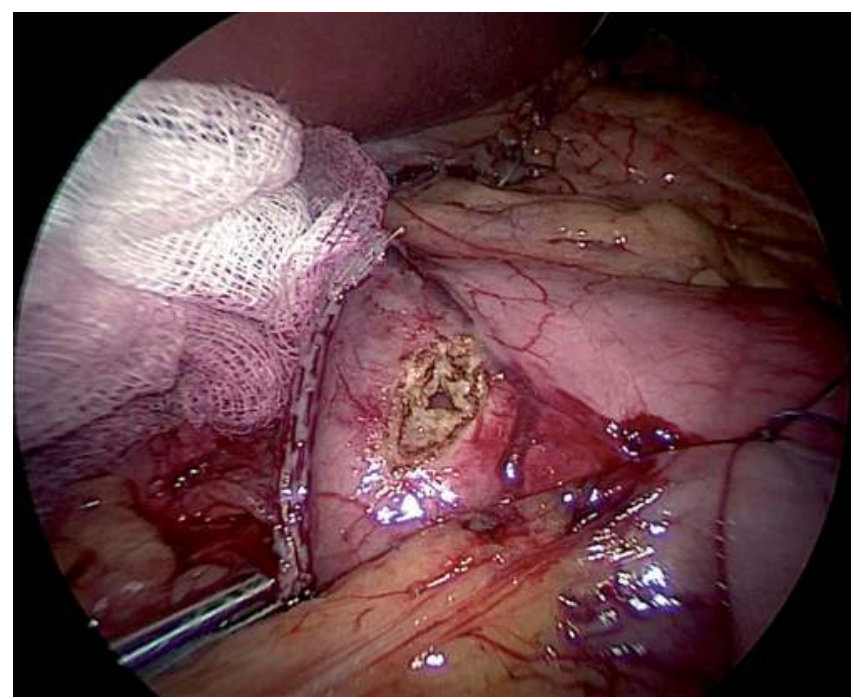

Figure 22. Duodenal enterotomy placed obliquely.

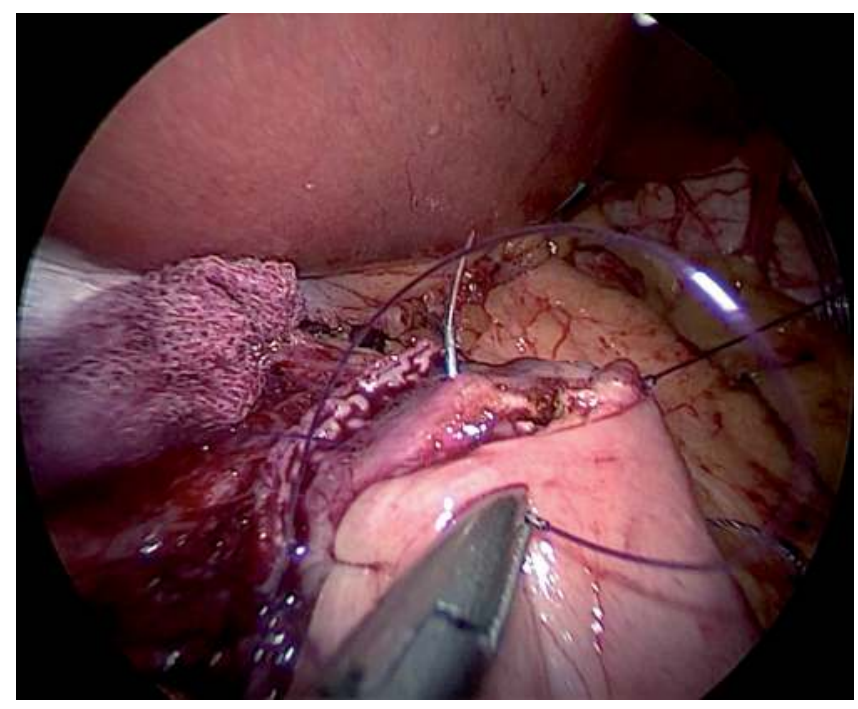

Figure 23. Completely hand-sewn duodenojejunal bypass.

\subsubsection{Postoperative care and follow-up}

Adhering to the early recovery after surgery (ERAS) postoperative protocol, adequate pain control is administered via the intravenous route and early ambulation is encouraged in our patients as early as six hours after surgery in order to avoid pulmonary complications. 
Once the patient is fully awake, clear liquid diet as instructed by our dietitian is commenced. Intravenous antibiotic is administered for one more day. Patients are usually discharged 2-3 days after surgery. Patients are placed on bariatric diet as instructed by the dietitian. Proton pump inhibitors are given for 1 month after surgery. Follow-up schedule is as follows: 1 week, $1,3,6$ and 12 months after surgery. One year after the surgery, patients are advised to followup every 6 months thereafter.

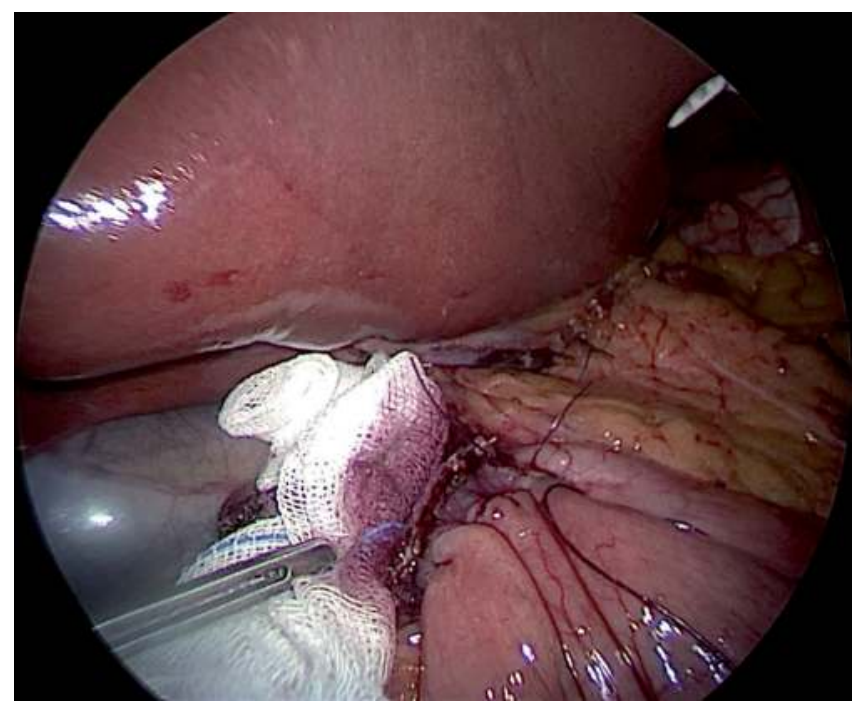

Figure 24. Anterior wall of DJB.

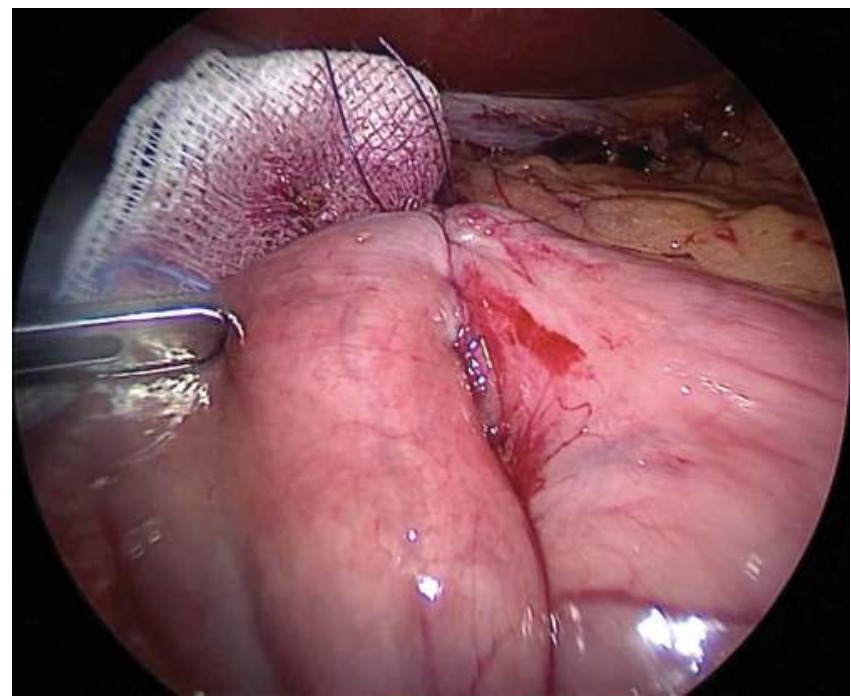

Figure 25. Posterior wall of DJB. 


\subsubsection{Surgical Outcomes}

The mechanism of weight loss in LDJB-SG is due to the resection of Ghrelin-secreting cells located in the fundus of the stomach. A significant drop in this hormone's level after the procedure causes decrease in sensation of hunger and early satiety. Furthermore, aside from its weight-loss effect, caloric restriction also helps in the improvement of insulin resistance and

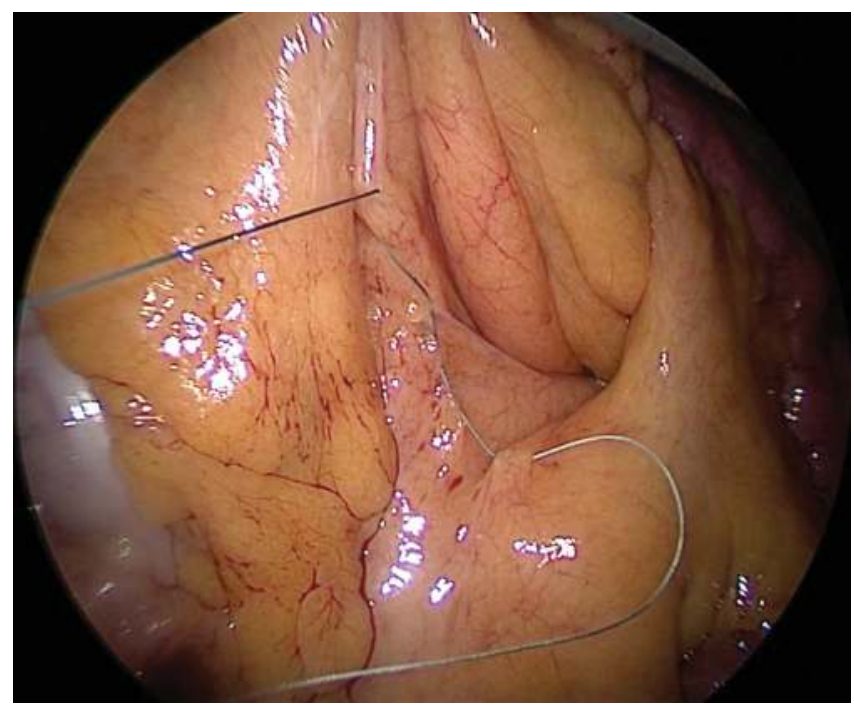

Figure 26. Closure of Petersons defect.

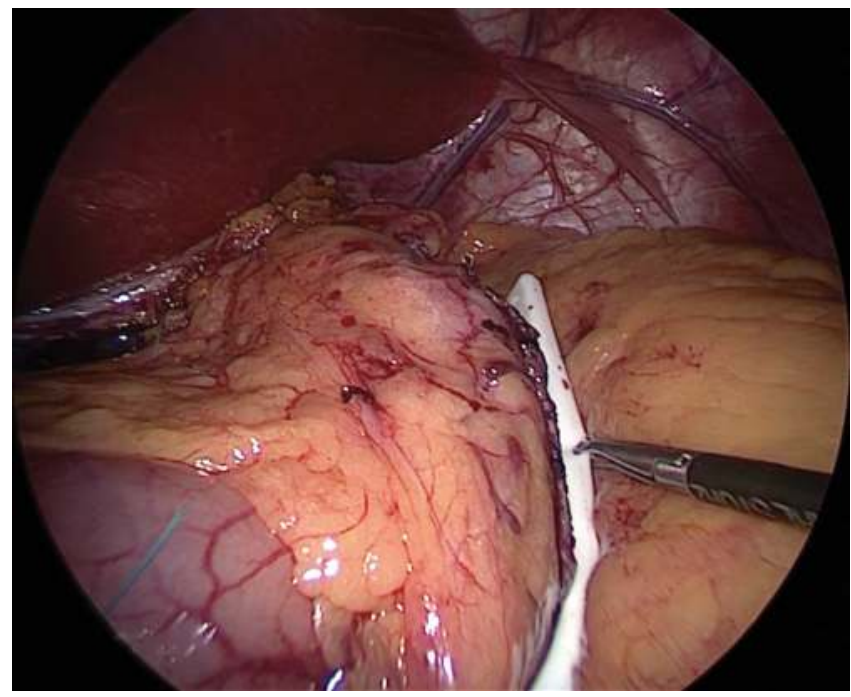

Figure 27. Placement of JP drain along DJB anastomosis and entire length of the sleeve suture line. 
increase in glucose tolerance. However, the efficient glycemic control of this procedure is predominantly due to the exclusion of the duodenum (Foregut theory) and the faster delivery of undigested food and more concentrated bile to the distal small bowel (Hindgut theory) which ensures secretion of incretins.

In a prospective study by Huang et al. published in 2013 comparing LDJB-SG with RYGB, LDJB-SG had a superior remission rate of T2DM (60\% vs $49 \%$ ) and a better over-all glycemic control (90\% vs 71\%) compared to RYGB in patients with BMI $\leq 35 \mathrm{~kg} / \mathrm{m}^{2}$ at 1 year after surgery. Fasting blood sugar levels was also significantly lower in the LDJB-SG group $(98.0 \pm 18.0$ vs $106.0 \pm 31.7 \mathrm{mg} / \mathrm{dl})$. The drop in HbA1C was also lower in the LDJB-SG compared to the RYGB $(6.0 \pm 0.9$ vs $6.3 \pm 1.2)$ although this did not attain a statistically significant level $(P$ value $=0.442)$. Resolution of other obesity-related comorbid factors were also seen in LDJB-SG that was comparable to the RYGB group (Hypertension $=85.7 \%$ vs $88.2 \%$ and Dyslipidemia $=70 \%$ vs $76.6 \%$ resolution rates) [8].

In a case-matched study comparing of 30 patients undergoing LDJB-SG and 30 patients undergoing RYGB, the mean BMIs dropped significantly to $22.4 \mathrm{~kg} / \mathrm{m}^{2}( \pm 2.4)$ (range $18.4-27 \mathrm{~kg} / \mathrm{m}^{2}$ ) and $21.9 \mathrm{~kg} / \mathrm{m}^{2}( \pm 2.5)$ (range $17.7-26.5 \mathrm{~kg} / \mathrm{m}^{2}$ ) from preoperative values of $28.2 \mathrm{~kg} / \mathrm{m}^{2}( \pm 3.6)$ and $27.8 \mathrm{~kg} / \mathrm{m}^{2}( \pm 3.8)$ at 1 year after surgery $(\mathrm{p}<0.01)$. However, no statistical difference was seen between the two groups. HbA1c and fasting glucose were also significantly decreased 1 year after surgery compared to its preoperative value from $8.98( \pm 1.75 \%)$ to $6.52 \%( \pm 1.03)$ and 168.3 $( \pm 54.9 \mathrm{mg} / \mathrm{dL})$ to $106.5 \mathrm{mg} / \mathrm{dL}( \pm 28.2 \mathrm{mg} / \mathrm{dL})$. Comparing complete remission of T2DM in 1 year after surgery, the LDJB-SG had a remission rate of $36.6 \%$ of patients $(11 / 30)$ compared to $30 \%(9 / 30)$ in the RYGB group. As far as complications, the rate for early complication favors that of RYGB ( 1 case vs 4 cases). However, there is a note of a trend toward lower occurrence of late complication rate in LDJB-SG compared to RYGB (5 vs 8 cases, $\mathrm{p}<0.08$ ) [11].

Lee et al. compared single anastomosis duodenojejunal bypass with sleeve gastrectomy (SADJB-SG) with RYGB and minigastric bypass (MGB). The operation time was significantly longer in SADJB-SG compared to the other types of bypass (181.7 mins vs 160 mins for RYGB vs 120.1 mins for MGB; p < 0.01). During the interim follow up period at 1, 3, 6 and 12 months after surgery, the mean BMI dropped to $32.9 \pm 4.8,29.9 \pm 6.8,27.6 \pm 5.4$ and $25.9 \pm 4.6 \mathrm{~kg} / \mathrm{m}^{2}$, respectively. The percentage weight loss during these same follow-up periods were 15.1, 20.325 and 32.7\%. 12 months after surgery, comparing percent weight loss (\% WL) of the three procedures, the results showed a higher percent weight loss for SADJB-SG (32.7 $\pm 7.9 \%$ SADJB-SG vs $28.9 \pm 9.0 \%$ MGB vs $26.1 \pm 4.1 \%$ RYGB). Results also showed a superior percent excess weight loss for SADJB-SG $(80.3 \pm 24.8 \%)$ compared to the two bypass procedures $(68.6 \pm 58.2 \% \mathrm{MGB}$ and $63.4 \pm 31.8 \% \mathrm{RYGB})$. In the same report, T2DM was seen in $>80 \%$ of the subjects with a preoperative mean HbA1c level of $9.2 \%$. This value decreased to $6.1 \% 1$ year after surgery with a complete remission rate of T2DM in $64 \%$ of the patients [12].

In an attempt to evaluate the role of duodenal exclusion in glycemic control, a matched group study comparing LDJB-SG and SG alone was done. At 1 year after surgery, the LDJB-SG (26 patients) presented with a higher percent excess weight loss $(87.2 \pm 14.9 \%$ vs $67 \pm 27.0 \% ; \mathrm{p}=0.23)$ and a lower BMI $(23.9 \pm 2.2$ vs $26.1 \pm 3.7 ; \mathrm{p}=0.065)$ compared to the SG 
alone group (29 patients). As far as T2DM remission rate is concerned, the LDJB-SG had a $92.3 \%$ total glycemic control rate compared to $86.2 \%$ in the SG alone group. The mean reduction in the $\mathrm{HbA1c}$ level for the LDJB-SG was likewise higher compared to the SG group (2.8 vs $2.1 \% \mathrm{p}=0.45$ ) [13].

\subsection{Sleeve gastrectomy with proximal jejunal bypass (Figure 28)}

\subsubsection{Indication and contraindication}

The indications and contraindications of PJB-SG are the same as that of the conventional stand-alone sleeve gastrectomy. Furthermore, this can also be offered as a metabolic surgery for patients with BMI between 27.5 and 35 with type 2 Diabets.

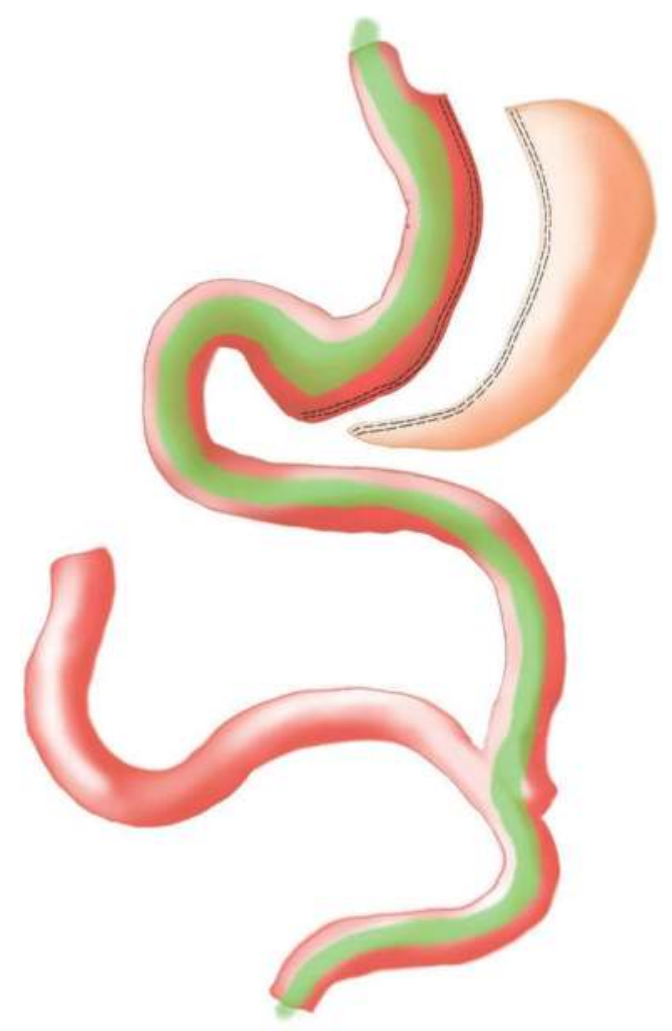

Figure 28. Schematic diagram of LPJB-SG.

\subsubsection{Preoperative workup and preparation}

Like in all bariatric procedures, a complete preoperative workup should be done in order to select appropriate candidates for the procedure. All patients are evaluated by a multidisciplinary 
team dedicated in bariatric surgery including a bariatric surgeon, bariatric physician, gastroenterologist, anesthesiologist, psychiatrist, nutritionist and fitness coach. Other specialists may be called upon if required.

\subsubsection{Positioning}

Patient is positioned supine with upper extremities abducted and lower extremities adducted. Patient is then secured to the operating table using straps and foot boards. Patient is placed in steep reverse Trendelenburg position.

\subsubsection{Operative technique}

\subsubsection{Port placement}

A 4-port technique is utilized with the surgeon positioned on the patient's right side and the assistant and camera man standing on the patient's left. Port placement is similar to LSG with an assistant port in left anterior axillary line below the costal margin (Figure 29).

\subsubsection{Pneumoperitoneum}

Pneumoperitoneum is accomplished either via Veress technique or through a $12 \mathrm{~mm}$ optical trocar using a 30-degree scope in the left periumbilical area.

\subsubsection{Placement of liver suspension tape}

Liver retraction is done using the LST similarly placed as in the standard LSG technique. Alternatively, a Nathanson liver retractor can also be used.

\subsubsection{Sleeve gastrectomy}

Standard sleeve gastrectomy is performed as previously described earlier in this chapter.

\subsubsection{Proximal jejunal bypass}

After completing the sleeve gastrectomy, ligament of Treitz is identified. Transection of the jejunum is done at $20 \mathrm{~cm}$ from the ligament of Treitz using either a $45 \mathrm{~mm}$ white cartridge reload (EGIA45AMT Endo GIA ${ }^{\mathrm{TM}} 45 \mathrm{~mm}$ articulating medium/thick reload with Tri-Staple Technology) or a $45 \mathrm{~mm}$ white cartridge reload (ECR45W Echelon Endopath ${ }^{\mathrm{TM}}$ tri-stapler reload cartridges by Ethicon). Next $300 \mathrm{~cm}$ of small bowel is measured distally and a sideto-side jejunojejunal anastomosis was fashioned out using the same cartridge and stapling device. The jejunojejunostomy is created with hand-sewn technique using 3-0 Absorbable Glyconate Monofilament running suture (3-0 B I BRAUN MONOSYNTM UNDYED 28" HR26 TAPER) (Figures 30-32).

\subsubsection{Closure of mesenteric defect}

The mesenteric defect is closed with simple running sutures using 2-0 nonabsorbable polyester suture (Ethibond Excel ${ }^{\mathrm{TM}}$ polyester suture). No drain is placed. 


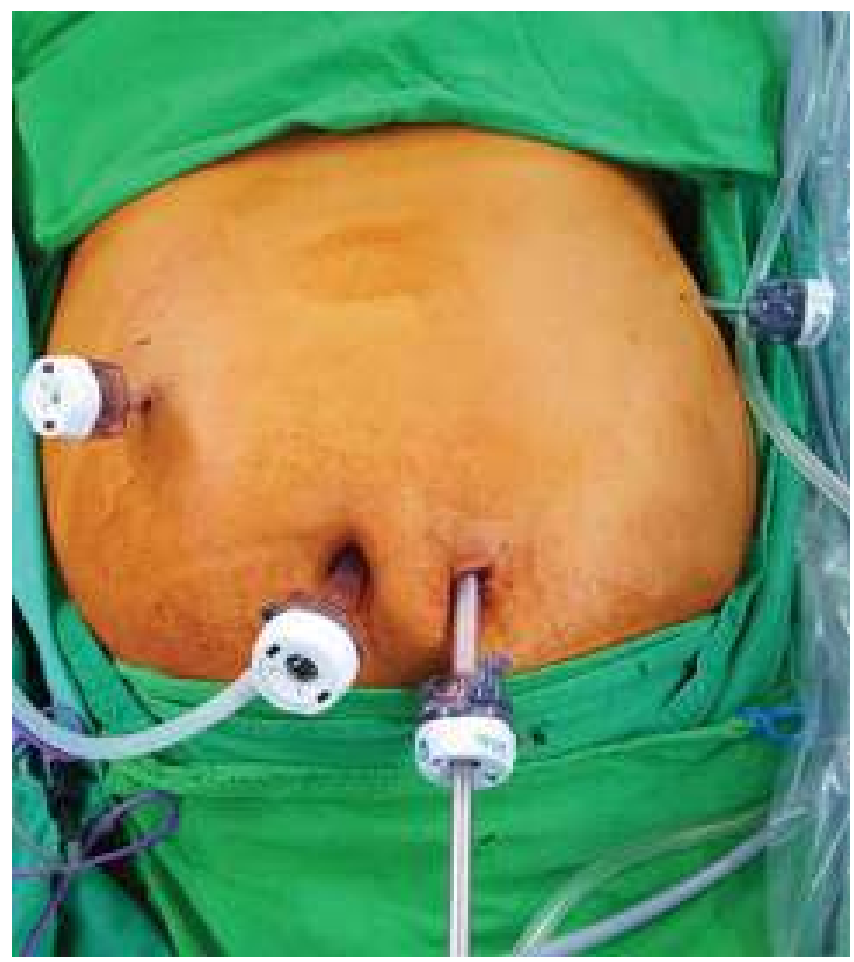

Figure 29. LPJB-SG port placement.

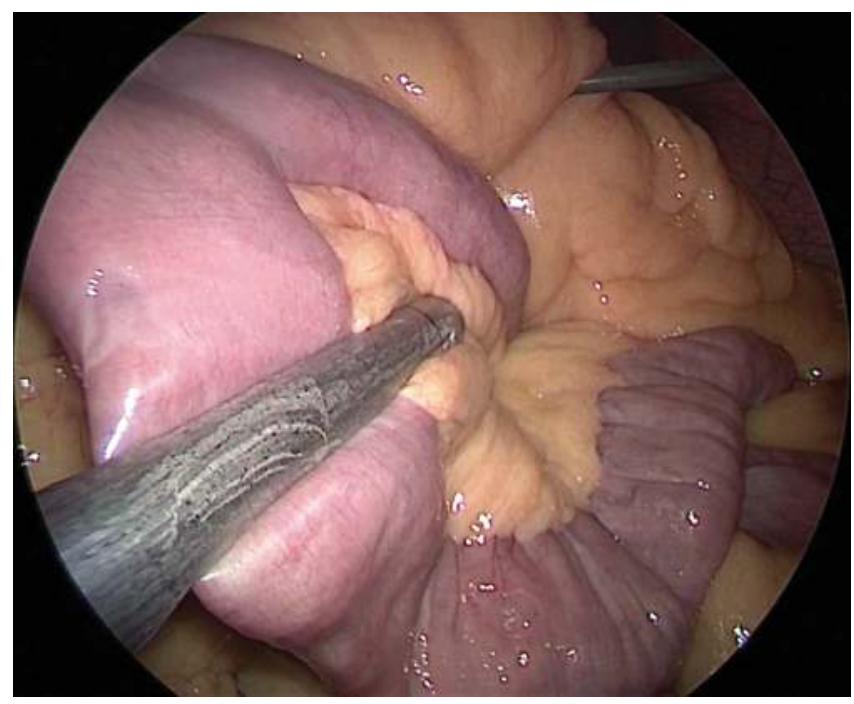

Figure 30. Transection of Bilio-pancreatic limb $20 \mathrm{~cm}$ from ligament of Treitz. 


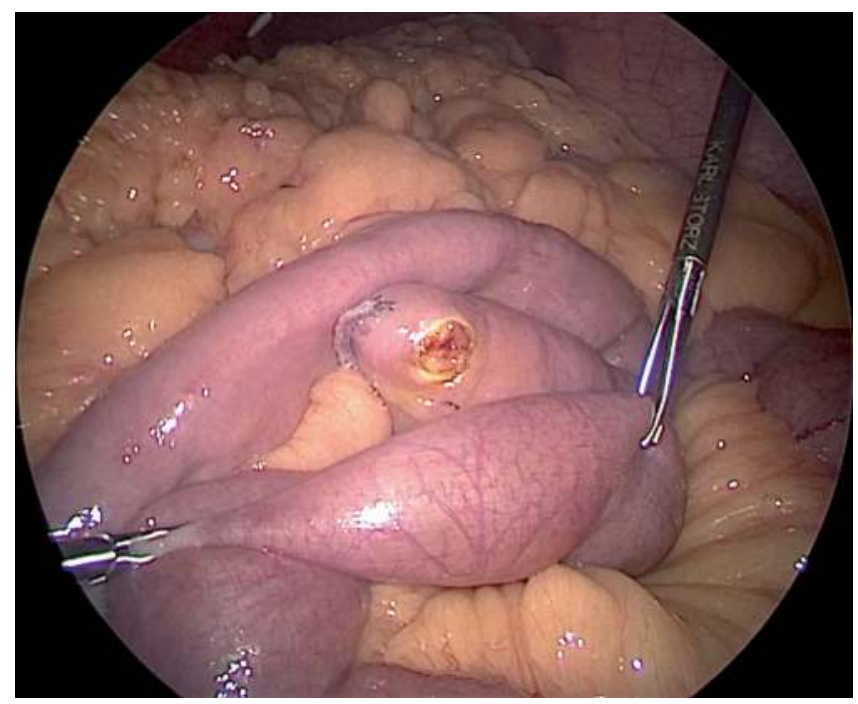

Figure $31.300 \mathrm{~cm}$ bypassed proximal jejunum.

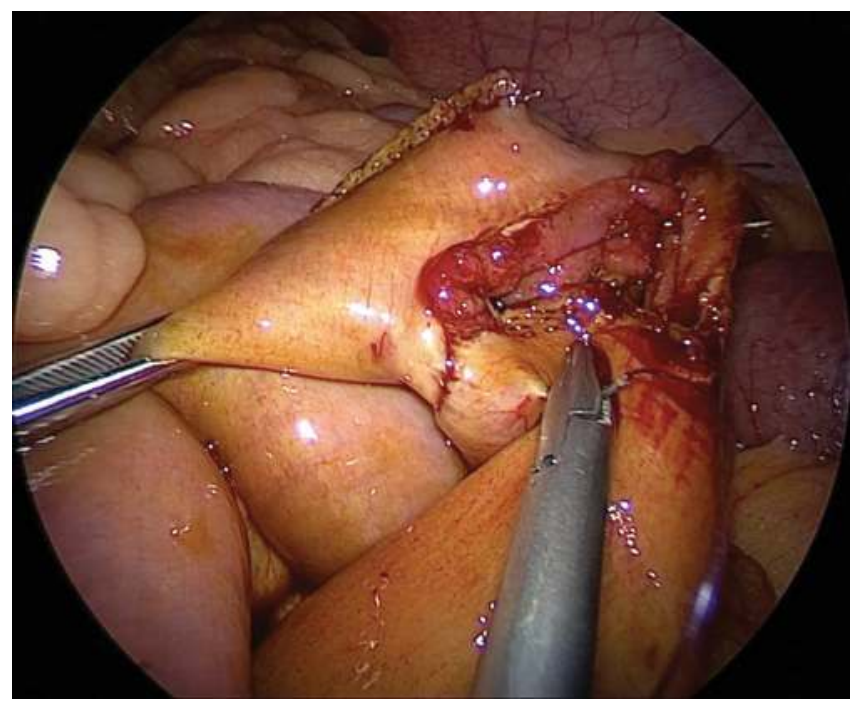

Figure 32. Closure of jejunojejunal anastomosis.

\subsubsection{Gastric sleeve fixation}

The suture line of the sleeved stomach is anchored with one or two stitches at the retroperitoneal fat using 3-0 Polyglactin 910 multifilament absorbable suture (J774D Ethicon 3-0 Coated Vicryl $^{\mathrm{TM}}$ Taper SH). This prevents inadvertent twisting of the tubularized stomach (Figure 33). 


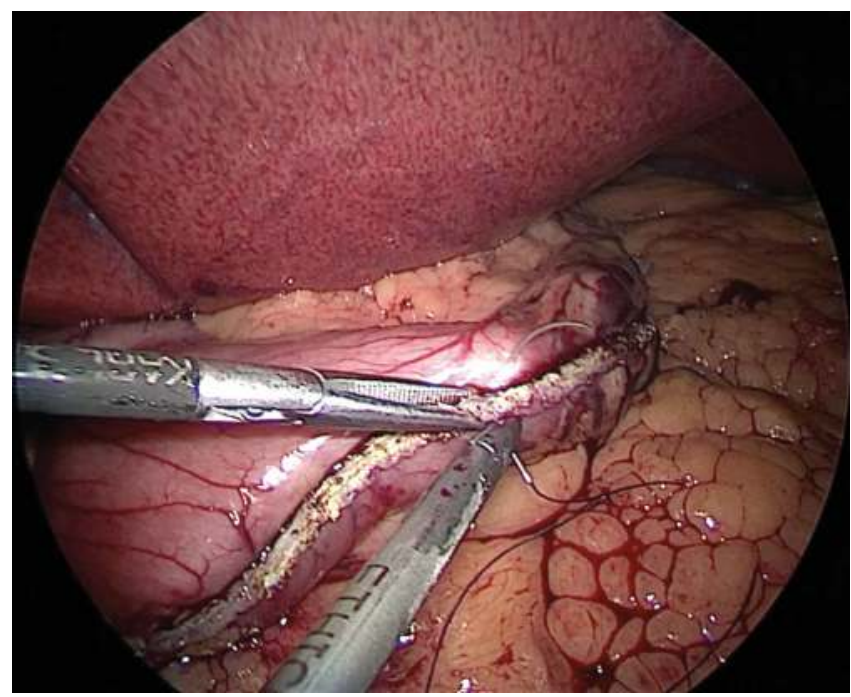

Figure 33. Anti torsion stitch.

\subsubsection{Surgical Outcomes}

Factors that contribute to the magnitude of weight loss and diabetic remission in sleeve-based procedures are still not fully elucidated. It probably owes its weight loss capabilities by eliminating the function of the stomach as a reservoir hereby reducing caloric content of processed food. Furthermore, because of the jejunojejunal bypass, the terminal ileum is exposed much earlier to undigested food and a more concentrated bile, both of which stimulate the release of incretins, primarily glucagon-like peptide 1 (GLP-1).

In the original study of Alamo et al., the average BMI and average weight at 12 months after vertical isolated gastroplasty with gastroenteral bypass were $23.4 \pm 3.3 \mathrm{~kg} / \mathrm{m}^{2}(19.2-27.7)$ and $65.1 \pm 15 \mathrm{~kg}$ (46-83) from $41.2 \pm 5.1 \mathrm{~kg} / \mathrm{m}^{2}(35.3-57.8)$ and $110.7 \pm 16.2 \mathrm{~kg}$. The mean percent excess weight loss (\% EWL) was $90.2 \pm 11.9 \%$ [3]. In other studies, the mean BMI was reduced to $21.4 \pm 1.9 \mathrm{~kg} / \mathrm{m}^{2}$ at 18 months' follow-up from $31.6 \pm 2.1 \mathrm{~kg} / \mathrm{m}^{2}$ with a mean percent EWL of $75.7 \pm 8.5 \%$. Comparing these figures with RYGB, in the study by Higa et al., where patients were followed-up for 10 years, the mean percent excess weight loss was $57 \%$ and the average postoperative BMI was $33 \pm 8.0 \mathrm{~kg} / \mathrm{m}^{2}$ at 10 years [14].

In a cohort study of 49 patients (2012) evaluating the efficacy of SGPJB in ameliorating T2DM in patients with BMI $<35 \mathrm{~kg} / \mathrm{m}^{2}, 81.6 \%$ (40/48) of patients with T2DM achieved complete remission after SGPJB with the remaining nine patients achieving improvement. As far as discontinuation of oral hyperglycemic agents and insulin dependence are concerned, $97.6 \%$ (40/41) of patients stopped taking their medications and $100 \%(8 / 8)$ stopped using insulin [10]. 


\section{Laparoscopic Roux-En-Y Gastric Bypass (Figure 34)}

Mason observed that distal gastrectomy with Billroth II reconstruction causes weight loss. The first open RYGB was performed for weight loss in 1967 while the first Laparoscopic RYGB was performed by Wittgrove [15]. Since then, laparoscopic RYGB is one of the most commonly performed bariatric procedure done for excess weight loss (Figure 34).

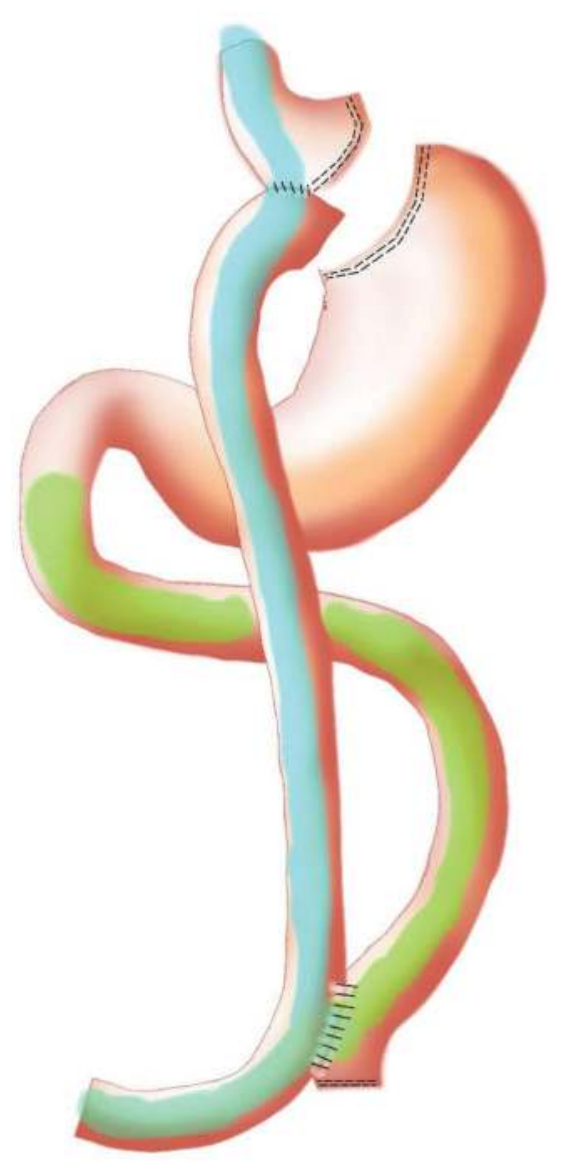

Figure 34. Schematic diagram of LRYGB.

Based on the 1991 NIH criteria, there are a number of widely accepted indication and contraindication which make a patient suitable for Bariatric or weight loss surgery:

- BMI > 40 without comorbidities.

- >35 with associated obesity-related illness such as diabetes or sleep apnea.

- Failed reasonable attempts at other weight loss techniques. 
- Obesity-related health problems.

- No psychiatric or drug dependency problems.

- A capacity to understand the risks and commitment associated with surgery.

- Pregnancy not anticipated in the first 2 years following surgery.

For Asians, BMI is reduced by 2.5 for the above.

\subsection{Operative technique}

\subsubsection{General preparations}

Preoperative investigation includes standard basic blood profile, thyroid function test, serum cortisol level, $\mathrm{HbA1c}$ and C-peptide if diabetic, whole abdominal ultrasound and upper gastrointestinal endoscopy. Patient will be placed on NPO and started with intravenous drip 125-150 ml/h. Anticoagulants are usually not needed but thromboelastic stockings are applied.

\subsubsection{Patient positioning}

Patient is positioned supine with both arms stretched out. Surgeon will be on the right side of the patient while the assistant surgeon and camera holder will be on the left. The assisting nurse will stand on the same side as the surgeon. Monitor(s) are placed at the head end of patient.

\subsubsection{Port placement}

A $12 \mathrm{~mm}$ optical port placed 3-finger breadth left lateral to umbilicus. A $5 \mathrm{~mm}$ port is placed 4-finger breadth to the right of umbilicus for surgeons left hand working port. A $12 \mathrm{~mm}$ port is placed at 45 degrees to right hand port in the right mid clavicular line for surgeon's left hand and stapler insertion. Another $5 \mathrm{~mm}$ port is inserted in upper left subcostal region in the anterior axillary line for assistant surgeon (Figure 35).

\subsubsection{Liver suspension tape}

Liver retraction is done with liver suspension tape using a straight needle prolene sutureJackson Pratt drain as previously described in this chapter for LSG.

\subsubsection{Creation of gastric pouch}

Gastric pouch is created by marking with orogastric calibration tube (OGCT) balloon with $25 \mathrm{ml}$ insufflation. Perigastric dissection is done with hook diathermy at lesser curve with preservation of hepatic branch of vagus nerve, usually after the first vascular branch. Twothree staplers (medium thickness) are used to create the gastric pouch. The final pouch size would be about 15-20 ml. Adhesion on the posterior aspect of gastric pouch are cleared. A small gastrostomy is created at the posterior side of the pouch with hook diathermy for the creation of gastrojejunostomy (Figures 36-42). 


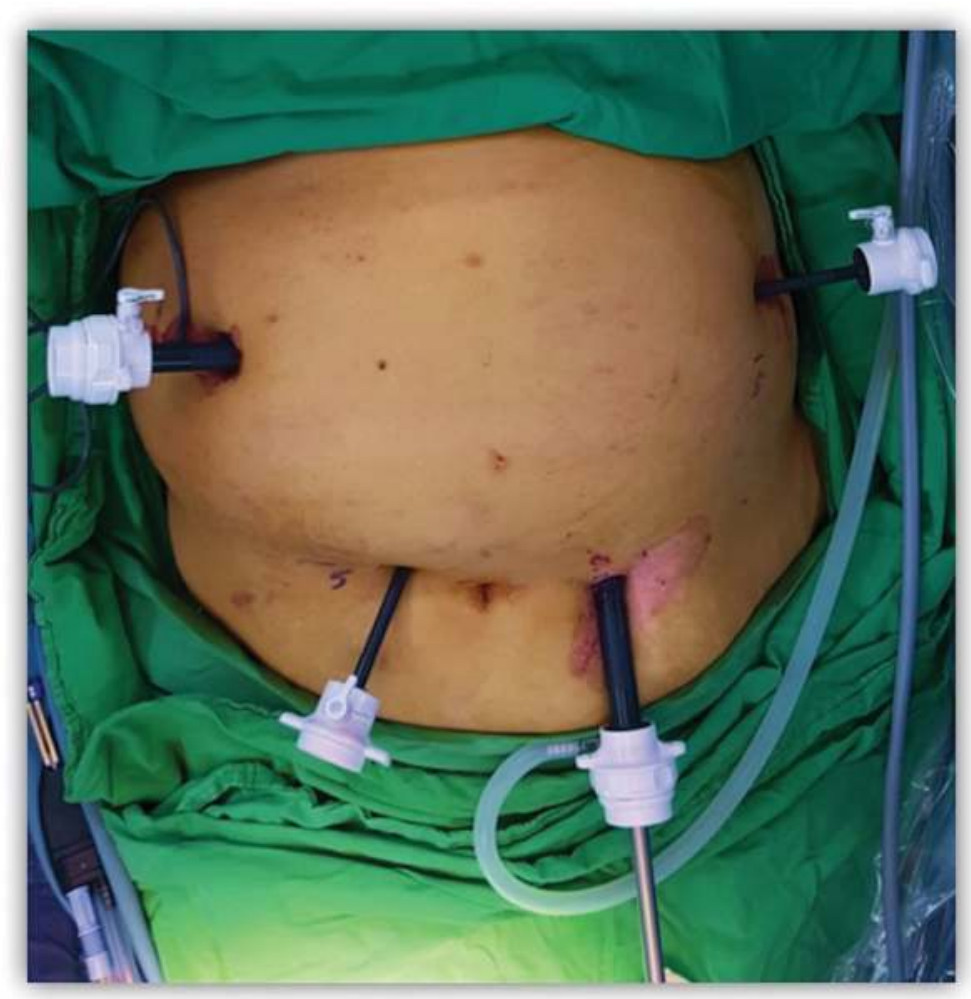

Figure 35. Port placement.

\subsubsection{Gastrojejunostomy}

Ligament of Treitz is identified and $100 \mathrm{~cm}$ of proximal jejunum is measured. Jejunotomy is created with hook cautery and loop of jejunum is anastomosed to the gastric pouch with linear stapler. Diameter of the gastrojejunal stoma is about $1.5-2 \mathrm{~cm}$. Jejunum is disconnected just proximal to gastrojejunostomy with a $45 \mathrm{~mm}$ white cartridge reload (EGIA45AMT Endo GIA ${ }^{\mathrm{TM}} 45 \mathrm{~mm}$ articulating thin reload with Tri-Staple Technology or ECR45W Echelon Endopath ${ }^{\mathrm{TM}}$ tri-stapler reload cartridges by Ethicon) to avoid creating a "candy cane" blind loop. Closure of gastrojejunal enterotomy is performed with Monocryl 3-0. 38 Fr orogastric tube is passed through the gastrojejunal anastomosis to check for patency (Figures 43-46).

\subsubsection{Jejunojejunostomy}

Jejunum is measured for a distance of $100 \mathrm{~cm}$ from gastrojejunostomy and side to side jejunojejunostomy is created with $45 \mathrm{~mm}$ cartridge (EGIA45AMT Endo GIA ${ }^{\mathrm{TM}} 45 \mathrm{~mm}$ articulating thin

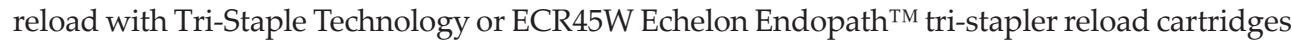
by Ethicon). Closure of jejunojejunal enterotomy is done with 3-0 Monocryl (Figures 47 and 48). 


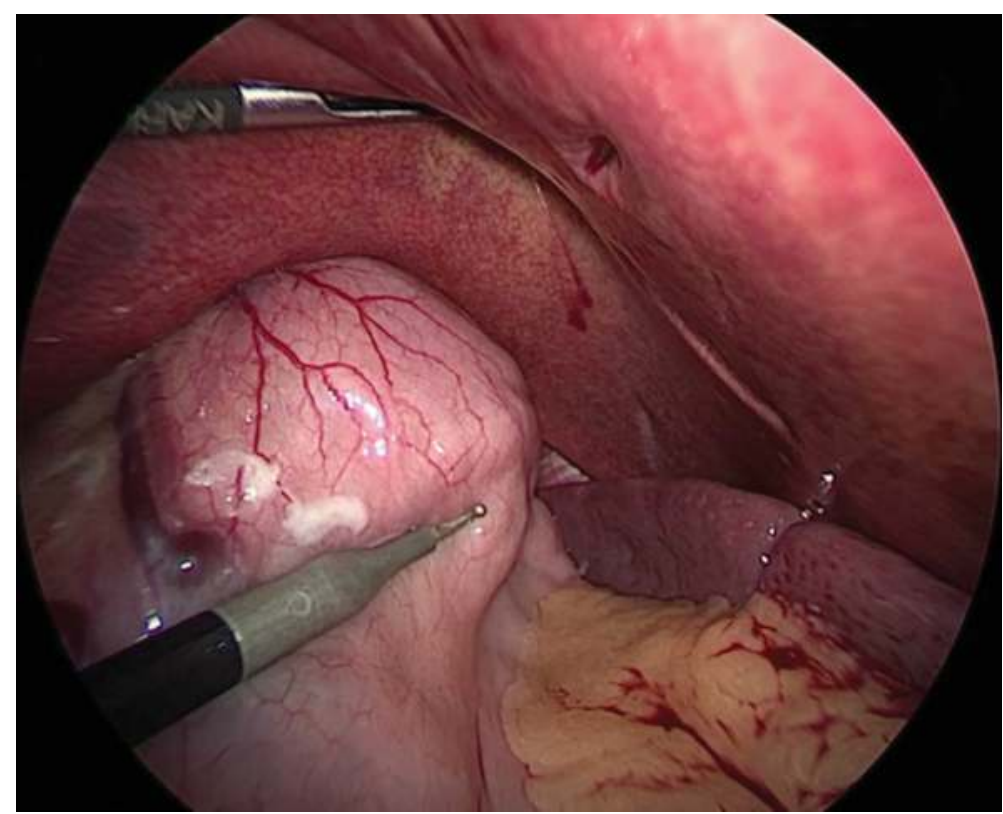

Figure 36. Calibration of gastric pouch.

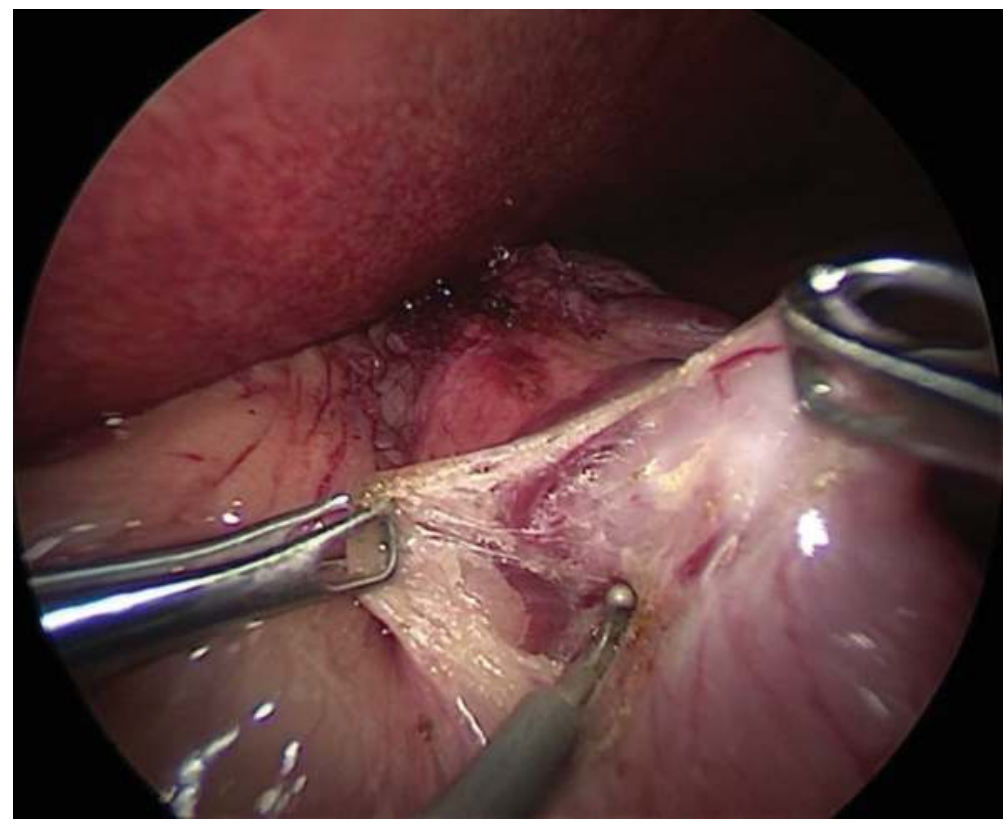

Figure 37. Perigastric dissection. 


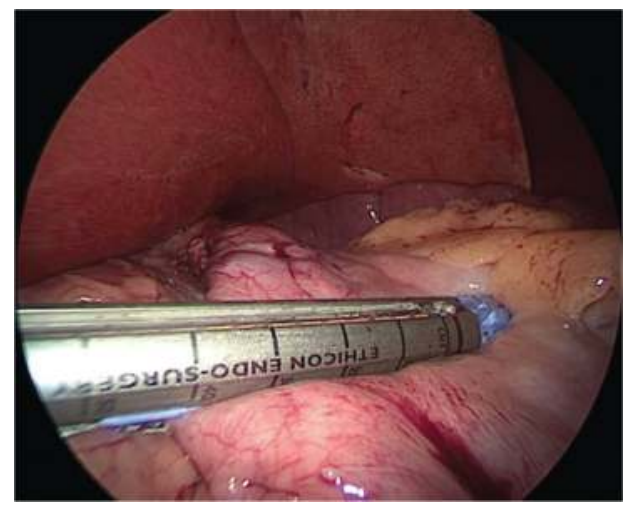

Figure 38. Firing of first staple.

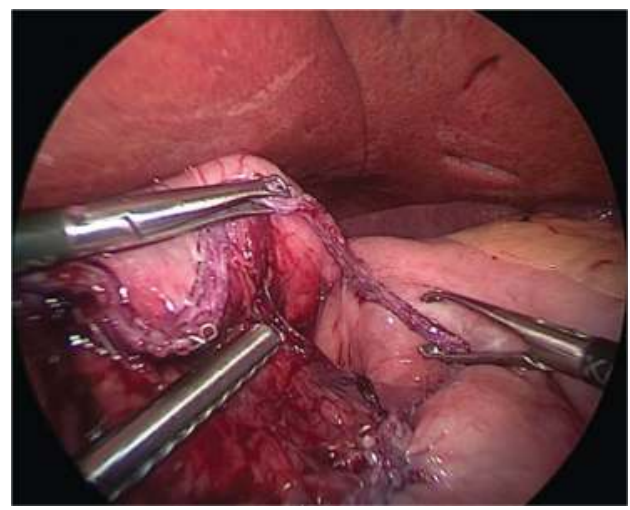

Figure 39. Dissection of adhesions at posterior gastric pouch.

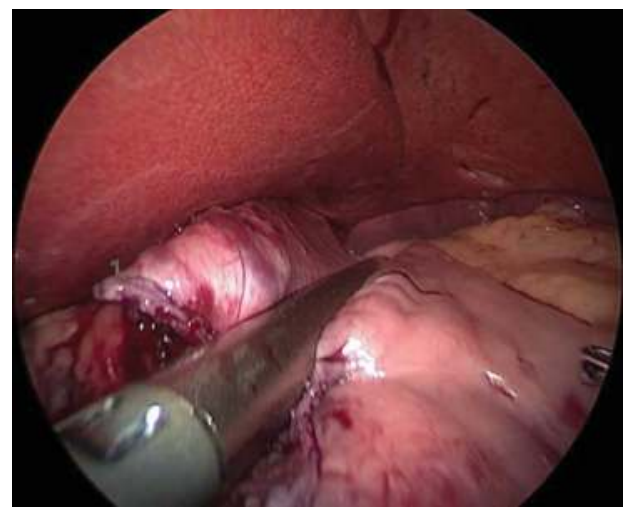

Figure 40. Firing of the 2nd stapler toward the angle of His. 


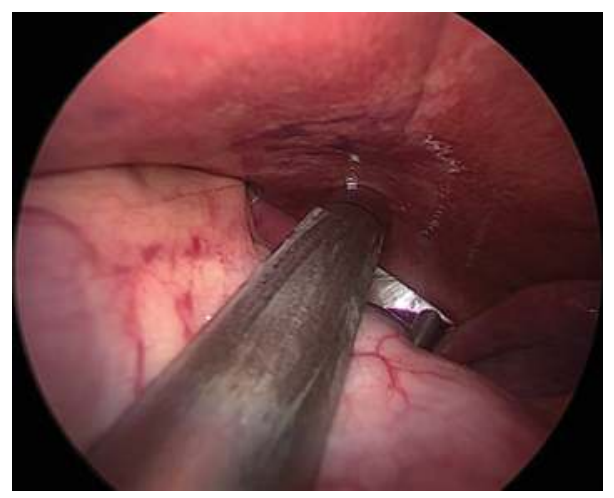

Figure 41. Firing of last stapler $1 \mathrm{~cm}$ away from GE junction.

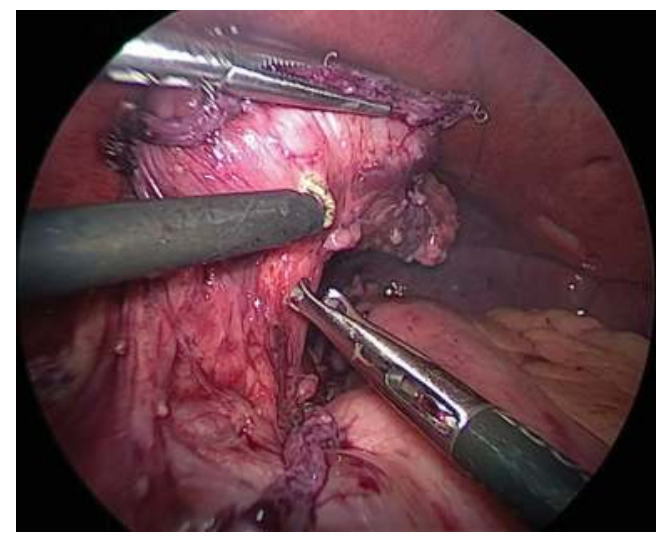

Figure 42. Creation of $1.5-2 \mathrm{~cm}$ gastrotomy.

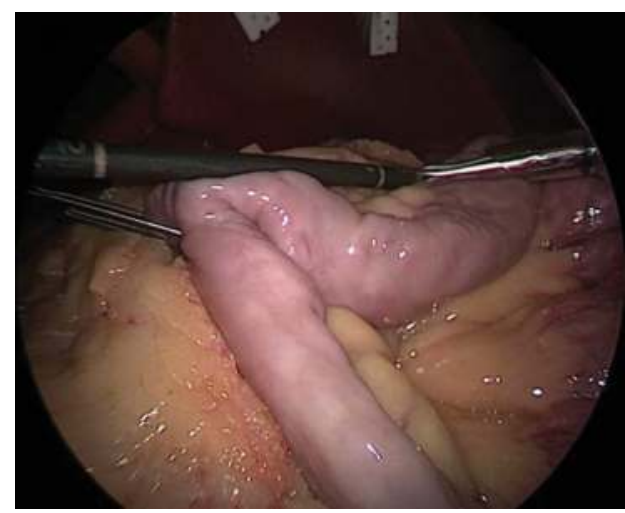

Figure 43. BP limb measuring 100-250 cm from the ligament of Treitz. 


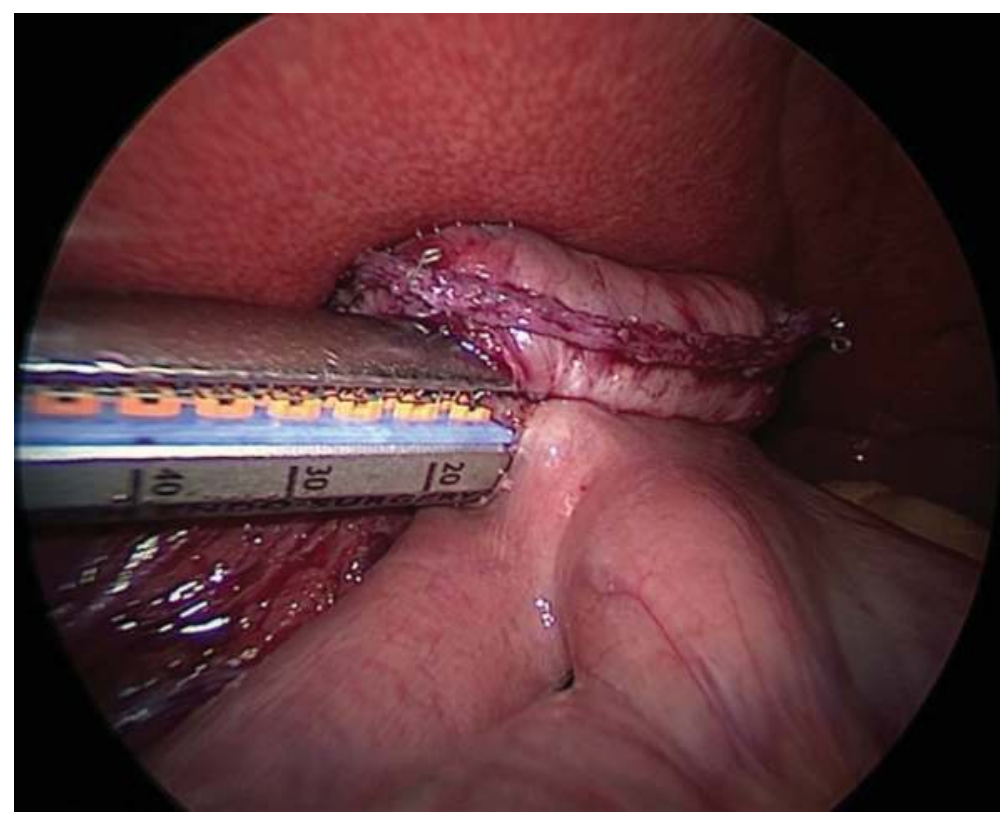

Figure 44. 1.5-2 cm side to side gastrojejunostomy.

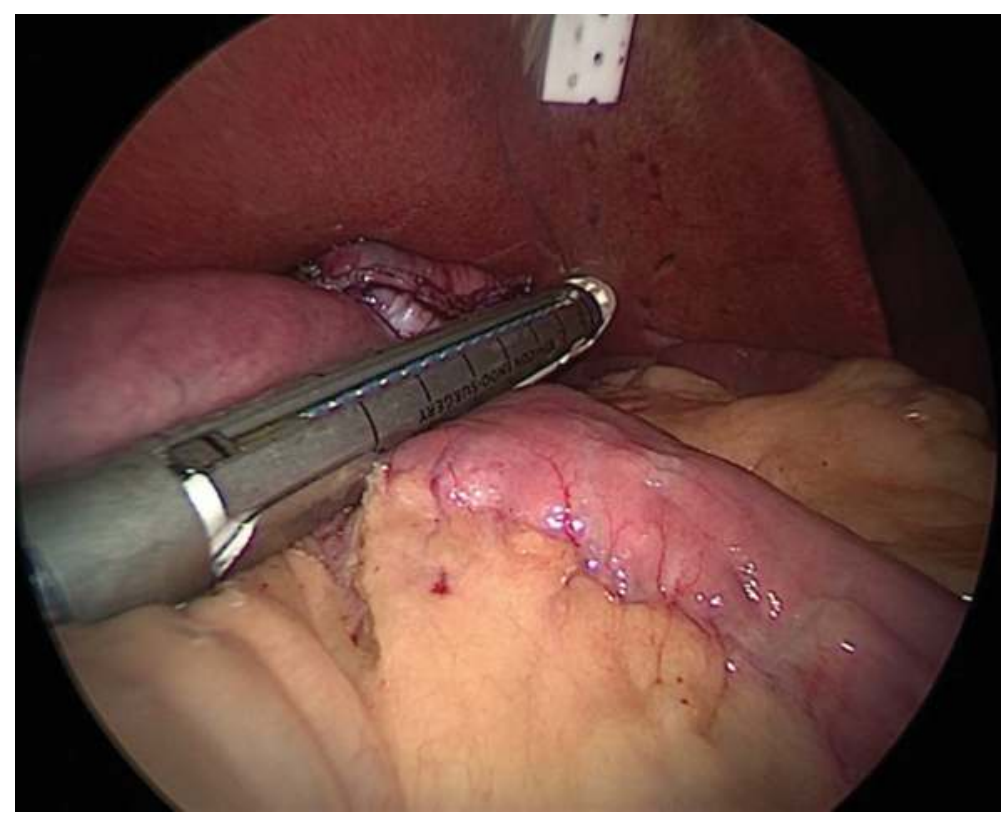

Figure 45. Transection of jejunum proximal to GJ anastomosis with thin stapler as close to GJ anastomosis as possible to avoid creating a candy cane stick blind loop. 


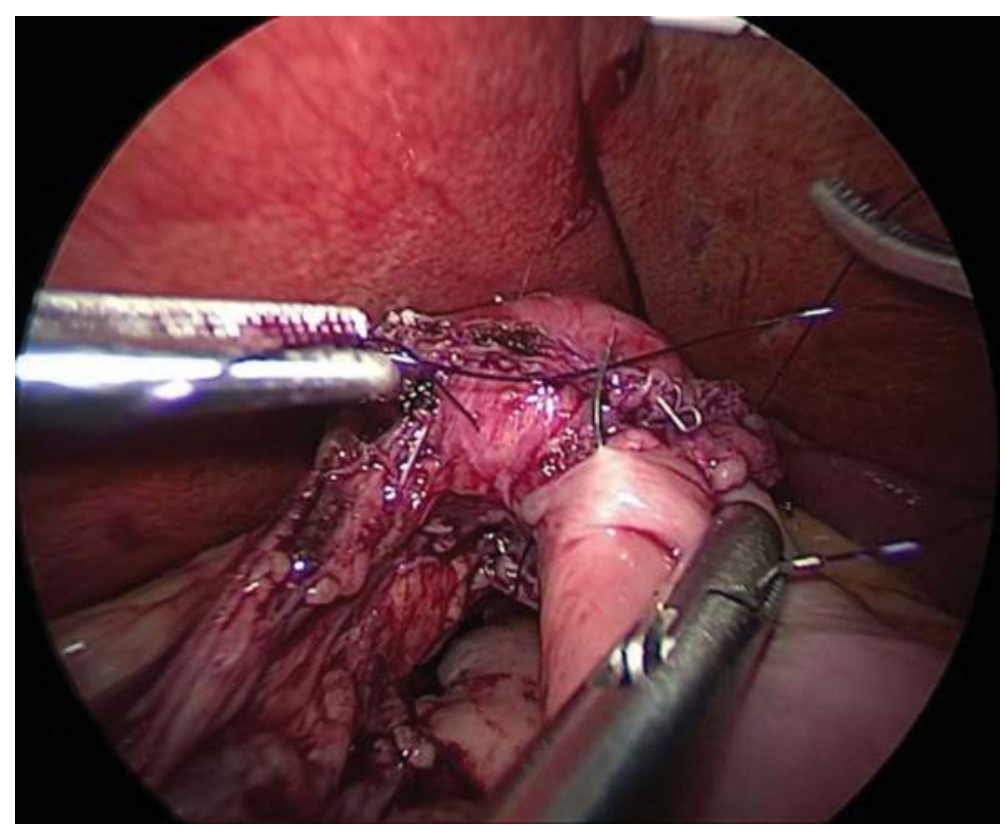

Figure 46. Gastrojejunal anastomosis completed by closure with single layer continuous suture using MONOSYN 3-0 round body needle.

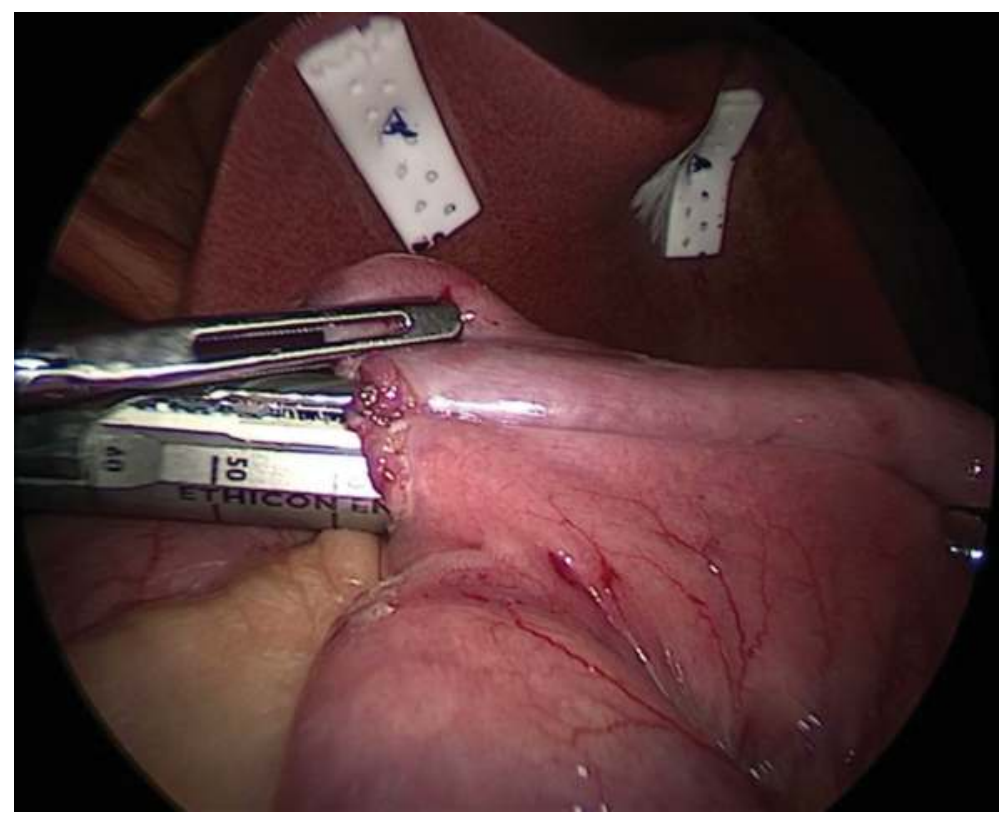

Figure 47. Side to side jejunojejunostomy. 


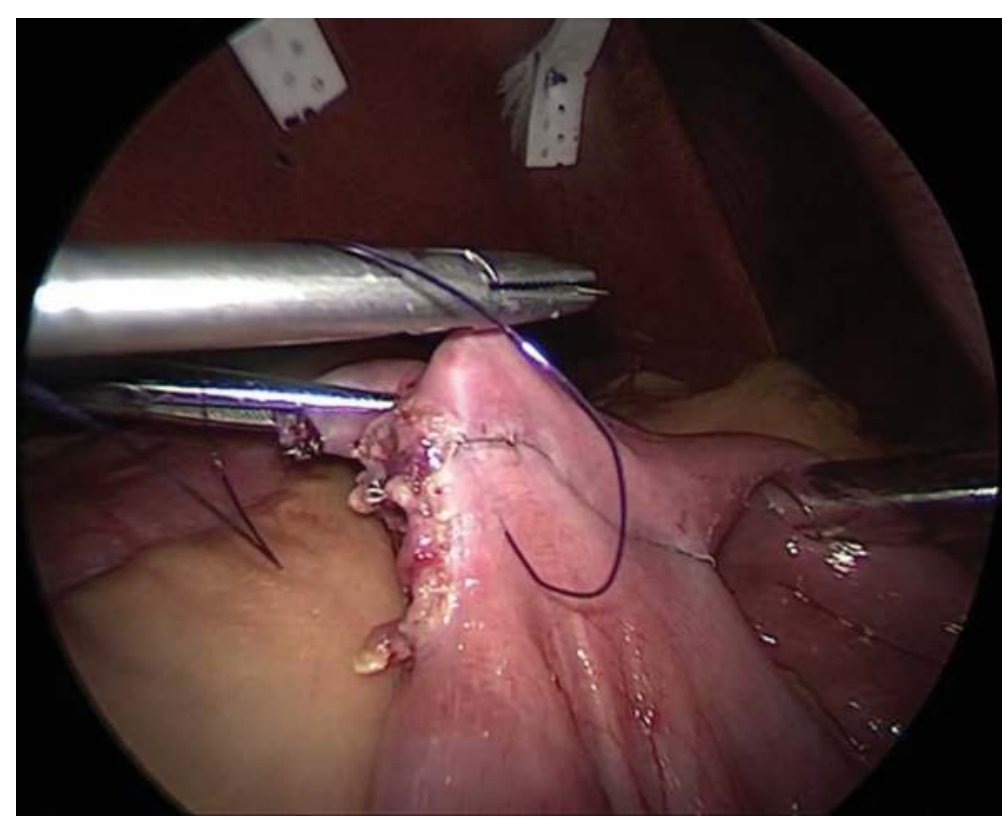

Figure 48. Hand-sewn closure of jejunojejunal anastomosis completed with single layer continuous suture using MONOSYN 3-0 round body needle.

\subsubsection{Closure of mesenteric defects}

Closure of mesenteric defects is performed with nonabsorbable sutures at jejunojejunal mesentery and Peterson defect (jejunotransverse mesocolon) to prevent internal herniation.

\subsubsection{Removal of liver suspension, hemostasis, deflation and closure}

After ensuring hemostasis at staple lines and anastomosis, liver suspension tapes are removed and hemostasis of liver punctures is done with diathermy.

\subsection{Surgical outcome}

\subsubsection{Excess weight loss}

Weight loss after bariatric surgery is dramatically seen in the first 12 months after surgery and continues at a slower rate up to 18 months postoperation. Excess weight loss after gastric bypass is between 72 and $82 \%$ within 12 months after surgery [16-18].

\subsubsection{Improvement of comorbidities}

Gastric bypass resulted in marked improvement in the biochemical markers of diabetes. Type 2 Diabetes Mellitus resolution is as high as $90.9 \%$ with mean fasting glucose reduction from 204 to $103 \mathrm{mg} / \mathrm{dl}$, mean $\mathrm{HbA} 1 \mathrm{c}$ reduction from 9.2 to $5.9 \%$ without T2DM medication in 12 months follow up. Up to $90 \%$ of patient did not need medication for control of glycaemia post operatively [19]. 
Gastric bypass can achieve a dramatic improvement of nonalcoholic fatty liver disease (NAFLD) both biochemically and histologically in morbid obesity [20]. Likewise, with significant weight loss and reduction of BMI after gastric bypass, pulmonary function improved in obese patients, which are correlated with decrease in waist circumference and possibly intraabdominal pressure [21].

\subsubsection{Complications}

Complications occur in up to $20 \%$ patients and include anastomotic leak $(0.25 \%)$, acute and late gastrojejuntostomy stricture $(5 \%)$, gastrojejunostomy hemorrhage $(1.5 \%)$, acute and late jejunojejunostomy stricture $(1.5 \%)$, iron deficiency anemia, marginal ulcers hemorrhage $(1.5 \%)$, gastric pouch dilatation $(1.5 \%)$, nonspecific abdominal pain, hair loss, internal herniation if mesenteric defects are not closed and rarely peroneal nerve palsy, acute cholecystitis, biliary stone with obstruction and cholangitis and intra-abdominal abscess [16-18].

\section{Author details}

Chih Kun Huang ${ }^{1 *}$, Sir Emmanuel S. Astudillo², Prasad M. Bhukebag ${ }^{1}$ and Khan Wei Chan ${ }^{1}$

*Address all correspondence to: dr.ckhuang@hotmail.com

1 Body Science and Metabolic Disorders International (B.M.I.) Medical Center, China Medical University Hospital, Taichung City, Taiwan

2 Center for Diabetes, Nutrition \& Weight Management, The Medical City Clark, Pampanga, Philippines

\section{References}

[1] Buchwald H, Avidor Y, Braunwald E, Jensen MD, Pories W, Fahrbach K, Schoelles K. Bariatric surgery: a systematic review and meta-analysis. JAMA. 2004;292(14):1724-1737.

[2] Rubino F, Nathan DM, Eckel RH, Schauer PR, Alberti KG, Zimmet PZ, Del Prato S, Ji L, Sadikot SM, Herman WH, Amiel SA, Kaplan LM, Taroncher-Oldenburg G, Cummings DE. Metabolic surgery in the treatment algorithm for type 2 diabetes: a joint statement by international diabetes organizations. Dia Care. 2016;39.6: 861-877. Web.

[3] Langer FB, Reza Hoda MA, Bohdjalian A, Felberbauer FX, Zacherl J, Wenzi E, Schindler K, Luger A, Ludvik B, Pregaer G. Sleeve gastrectomy and gastric banding: effects on plasma ghrelin levels. Obes Surg 2005;15(70):1024-1029.

[4] Himpens J, Dobbeleir J, Peeters G. Long-term results of laparoscopic sleeve gastrectomy for obesity. Ann Surg 2010;252 (2):319-324.

[5] Basso N, Leonetti F, Mariani P, et al. Early hormonal changes after sleeve gastrectomy in diabetic obese patients. Obes Surg. 2010;20(1):50-55. 
[6] Arman GA, Himpens J, Dhaenens J, et al. Long-term (11+years) outcomes in weight, patient satisfaction, comorbidities, and gastroesophageal reflux treatment after laparoscopic sleeve gastrectomy. Surg Obes Relat Dis 2016. [Epub ahead of print]. 10.1016/j. soard.2016.01.013

[7] Huang CK, Goel R, Tai CM, et al. Novel metabolic surgery for type 2 diabetes mellitus: loop duodenojejunal bypass with sleeve gastrectomy. Surg Laparosc Endosc Percutan Tech 2013 Dec;23(6):481-485.

[8] Huang CK, Ahluwalia JS, Garg A, Taweerutchana V, Ooi A, Chang PC, Hsin MC, et al. Novel Metabolic/Bariatric Surgery - Loop Duodenojejunal Bypass with Sleeve Gastrectomy (LDJB-SG). Essential and Controversies in Bariatric Surgery. 2014 Chapter 7: 133-144.

[9] Alamo MA, Torres CS, Perez LZ, et al. Vertical isolated gastroplasty with gastro-enteral bypass: preliminary results. Obes Surg. 2006;16:353-358.

[10] Alamo M, Sepulveda M, Gellona J, Herrera M, Astorga C, Manterola C, et al. Sleeve gastrectomy with jejunal bypass for the treatment of type 2 diabetes mellitus in patients with body mass index $<35 \mathrm{~kg} / \mathrm{m}^{2}$ : a cohort study. Obes Surg. 2012;22:1097-1103.

[11] Huang CK, Tai CM, Chang PC, Malapan K, Tsai CC, Yolsuriyanwong K et al. Loop duodenojejunal bypass with sleeve gastrectomy: comparative study with Roux-en-Y gastric bypass in type 2 diabetic patients with a BMI $<35 \mathrm{~kg} / \mathrm{m}^{2}$, first year results. Obes Surg. 2016;26(10):1-11.

[12] Lee WJ, Lee KT, Kasama K, Seike Y, Ser KH, Chun SC, Chen JC, Lee YC, et al. Laparoscopic single-anastomosis duodenal-jejunal bypass with sleeve gastrectomy (SADJB-SG): short-term result and comparison with gastric bypass. Obes Surg. 2014;24:109-113.

[13] Lee WJ, Almulai AM, Tsou JJ, Ser KH, Lee YC, Chen SC, et al. Duodenal-jejunal bypass with sleeve gastrectomy versus the sleeve gastrectomy procedure alone: the role of duodenal exclusion. Surg Obes Relat Dis. 2015 Jul-Aug; 11(4):765-770.

[14] Higa K, Ho T, Tercero F, Yunus, T, Boone K, et al. Laparoscopic Roux-en-Y gastric bypass: 10-year follow-up. Surg Obes Relat Dis. 2011;7:516-525.

[15] Wittgrove, et al. Laparoscopic gastric bypass, Roux-en-Y: preliminary report of 5 cases. Obes Surg. 1994;4:353-357.

[16] Huang, et al. Laparoscopic Roux-en-Y gastric bypass for morbidly obese Chinese patients: learning curve, advocacy and complications. Obes J. 2008; 18:776-781.

[17] Huang, et al. Use of individual surgeon versus surgical team approach: surgical team outcomes of laparoscopic Roux-en-Y gastric bypass in an Asian medical center. Surg Obes Relat Dis. 2012;8:214-219.

[18] Sjostrom L. Review of the key results from the Swedish obese subjects (SOS) triala prospective controlled intervention study of bariatric surgery. J Inter Med. 2013; 273(3):219-34. 
[19] Huang, et al. Laparoscopic Roux-en-Y gastric bypass for the treatment of Type II diabetes mellitus in Chinese patients with body mass index of 25-35. Obes Surg. 2011;21:1344-1349.

[20] Tai et al. Improvement of non-alcoholic fatty liver disease after bariatric surgery in morbidly obese Chinese patients. Obes Surg. 2012;22:1016-1021.

[21] Wei, et al. Surgically induced weight loss, including reduction in waist circumference, is associated with improved pulmonary function in obese patients. Surg Obes Relat Dis. 2011;7:599-604. 
